\title{
Wave diffraction and radiation by a vertical circular cylinder standing in a three- dimensional polynya
}

\author{
K. Ren ${ }^{a}$, G.X. Wu ${ }^{a}{ }^{\dagger}$, C.Y. Ji ${ }^{b}$ \\ ${ }^{a}$ Department of Mechanical Engineering, University College London, Torrington Place, London WC1E 7JE, \\ United Kingdom \\ ${ }^{b}$ School of Naval Architecture and Ocean Engineering, Jiangsu University of Science and Technology, \\ Zhenjiang 212003, China
}

† Corresponding author: G.X.Wu (g.wu@ucl.ac.uk)

\section{Abstract}

The wave diffraction and radiation problem of a body in a polynya surrounded by an ice sheet extending to infinity is considered through a vertical circular cylinder. The ice sheet is modelled through the elastic thin-plate theory and the fluid flow through the linearized velocity potential theory. In particular, when the polynya is of the circular shape, eigenfunction expansion method is applied to the two regions below the ice sheet and the free surface respectively, and the velocity and pressure continuity conditions are imposed on the interface of the two regions. The wave motion in the polynya, the hydrodynamic coefficients as well as the exciting forces on a body located arbitrarily in the polynya are calculated. The nature of highly oscillatory behaviour of the results is investigated and their physical implications are discussed.

\section{Keywords:}

three-dimensional polynya; ice sheet, vertical cylinder; wave diffraction/radiation; eigenfunction expansion method. 


\section{Introduction}

Interaction of water waves with an offshore platform is always a major concern in the ocean engineering. The loading on the structure and its response, or the motion excited by the wave have serious implications to its safety as well as its smooth and efficient operation. Therefore, there has been continuing effort to improve the understanding of the nature of wave interaction with a platform. Typical earlier work on this problem includes Molin (1979), Lighthill (1986), Eatock Taylor \& Hung (1987a), Kim \& Yue (1988), Eatock Taylor \& Sincock (1989), Faltinsen (1990), Chen et al. (1995) and more recent ones include that by Zhou \& Wu (2015).

A typical platform usually has a number of cylindrical legs. Thus, wave interactions with circular cylinders have been receiving considerable interests. Drake et al. (1984) considered an articulated cylinder and both the linear force and drift force were calculated. Eatock Taylor \& Hung (1987b) studied the second-order diffraction force on a cylinder in regular waves. Eatock Taylor et al. (1989) provided an analytical solution for the second-order wave pressure on a vertical circular cylinder without obtaining the complete solution of the secondorder problem. Later, Chau \& Eatock Taylor (1992) obtained the complete solution of the second-order diffraction problem of a vertical circular cylinder in regular waves. Huang \& Eatock Taylor (1996) considered the second-order diffraction problem of a truncated circular cylinder in regular waves. Bai \& Eatock Taylor (2007) studied the wave diffraction by a vertical circular cylinder by the fully nonlinear theory. Research work on multiple cylinders were conducted by Eatock Taylor \& Hung (1985), Linton \& Evans (1990), Maniar \& Newman (1997), Malenica et al. (1999) in open water, and by Linton \& McIver (1996), Evans \& Porter (1997), Ma et al. (2001) and Wang \& Wu (2010) in a tank or channel. 
The work mentioned above is on a structure in the open sea with its surface extending to infinity. The latest development in the arctic engineering has led to some new challenges. The large ice sheet has significant effect on the wave propagation and diffraction (Fox \& Squire, 1994). The present work considers a cylindrical structure in a polynya surrounded by an ice sheet extending to infinity. The aim is to shed some lights into the nature of wave/ice/body interaction in the three-dimensional problems.

There has a large body of work on wave/ice interaction. A summary of earlier work was given by Squire et al. (1995) and more recent reviews were given by Squire $(2007,2011)$, covering ocean waves interaction with the continuous ice sheets and with the marginal ice zone (MIZ). The linear elastic thin-plate theory is commonly adopted in these problems for the ice. The fluid is usually assumed to be inviscid and incompressible, and the flow to be irrotational. The interaction between the ice sheet and the wave is accounted for through the dynamic and kinetic conditions based on the pressure and normal velocity.

The problem of wave interaction with continuous ice sheets has been receiving a considerable amount of interest in various contexts. Fox \& Squire $(1990,1994)$ considered the wave propagating normally and obliquely into a semi-infinite ice sheet by adopting the eigenfunction expansion method. Similar problems were considered by Sahoo et al. (2001) by introducing an inner product with orthogonality. In addition to the eigenfunction expansion method, the WienerHopf method has been often used for such a problem, for example by Balmforth \& Craster (1999), Tkacheva $(2001,2004)$ and Chung \& Fox (2002). Meylan \& Squire (1994) constructed a Green's function to convert the governing equation an integral equation and solve the problem of wave interaction with a solitary ice floe as well a pair of ice floes. Meylan \& Squire (1996) considered a solitary 
circular ice floe floating on open water surface. Wang \& Meylan (2004) developed a higher-order numerical scheme by the coupling boundary element and finite element method to study the wave interaction with an elastic plate of arbitrary shape. Andrianov \& Hermans (2005) used a Green's function technique to investigate the wave diffraction by an elastic plate floating on water of both infinite depth and finite depth. For the three-dimensional polynya problem, Bennetts \& Williams (2010) solved the water wave scattering by a polynya of arbitrary shape in an infinite floating ice sheet via a Galerkin technique numerically. Later, Smith et al. (2011) investigated a related problem of wave scattering by an arbitrary shaped cavity in an elastic thin plate.

The work listed above is mainly about interaction of wave with ice sheet. When a body is present, Sturova (2015) considered a two-dimensional horizontal cylinder submerged in polynya while Ren et al. (2016) and Li et al. (2018) considered a floating body. For the three-dimensional diffraction problem, Malenica \& Korobkin (2003) investigated the interaction between waves and a vertical cylinder clamped by a finite ring-shaped ice sheet. Brocklehurst et al. (2011) considered the hydroelastic wave diffraction by a vertical cylinder being frozen into an ice sheet of infinite extent.

It appears there has been hardly any work on the three-dimensional problem of wave/ice sheet/floating body interaction. This is the purpose of the present work. We consider wave diffraction and radiation by a vertical circular cylinder arbitrarily standing in a circular-shaped polynya. The linear velocity potential is used. The solution procedure is based on the method of matched eigenfunction expansions in two regions below the water surface and ice sheet respectively. The kinematic and dynamic continuity conditions are imposed on the interface of the two regions. In the following sections, the mathematical procedure will be first discussed, followed by the detailed solution method. The hydrodynamic 
coefficients, and wave exciting forces are obtained through the near field and farfield equations. Results are also provided for the wave run-ups on the ice edge. The nature of interactions between wave/ice sheet/body is analysed and the implications are discussed.

\section{Mathematical Formulations}

The problem of wave diffraction and radiation by a bottom-mounted vertical circular cylinder with radius $a$ standing in a circular domain of open water confined by an ice sheet extended to infinity is sketched in Fig.1. A Cartesian coordinate system $(x, y, z)$ is established with its origin at the centre of the circular open water area and on the mean free surface. Its $x$-axis is along the propagation direction of the incident wave and its $z$-axis points upwards. A cylindrical coordinate system $(r, \theta, z)$ is also established with the same origin and $(x, y)=(r \cos \theta, r \sin \theta)$. A similar cylindrical coordinate system $\left(r_{1}, \theta_{1}, z\right)$ is defined with the vertical axis along the centre line of the cylinder, or its origin at $\left(r_{0}, \theta_{0}\right)$ in the previous cylindrical coordinate system. A long-crested flexural gravity wave comes from infinity, which will be scattered by the ice edge and the vertical cylinder. The ice sheet is assumed to have constant thickness $h$ and density $\rho_{\text {ice }}$. The radius of the circular open water area is $c$ and the water depth is $H$. The entire fluid domain is divided into two: domain $I$ below the open water surface and domain II below the ice sheet, as shown in Fig. 1. 


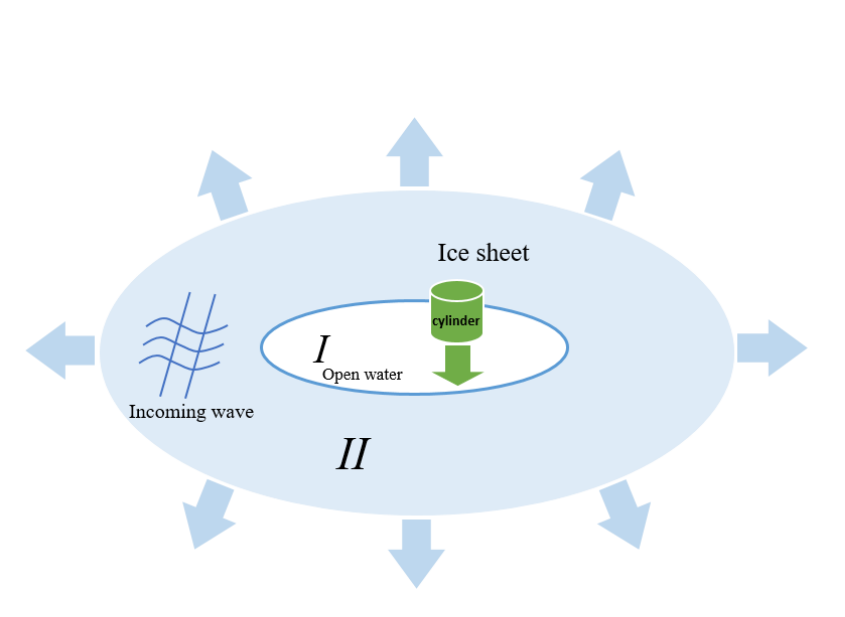

(a)

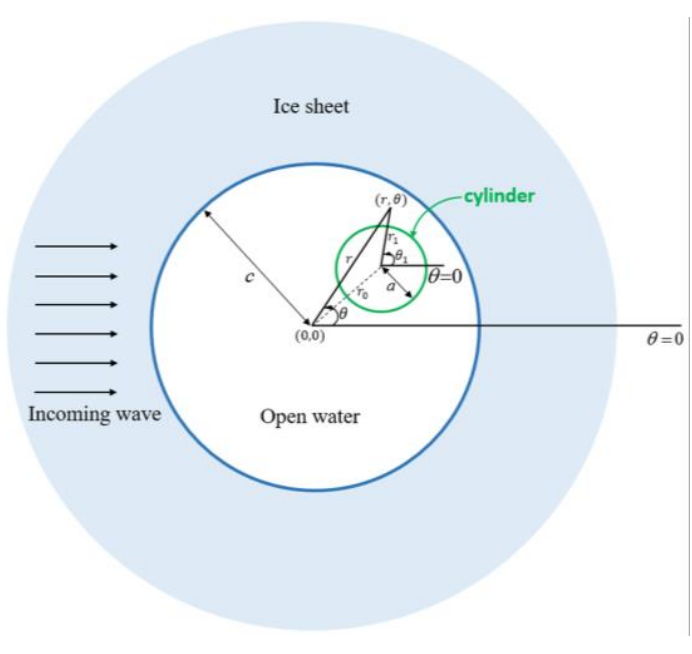

(b)

Figure 1. Sketch of the problem and cylindrical coordinate systems: (a). general view; (b) top view.

The fluid is assumed to be ideal, incompressible and its motion to be irrotational. The fluid flow can be described by the velocity potential, which is denoted as $\Phi(r, \theta, z, t)$. For a regular incoming wave of frequency $\omega$, the velocity potential for periodic motion can be written as

$$
\Phi(r, \theta, z, t)=\operatorname{Re}\left\{\left(\phi_{0}(r, \theta, z)-\sum_{j=1}^{6} i \omega \alpha_{j} \phi_{j}(r, \theta, z)\right) \times e^{-i \omega t}\right\}
$$

where $\phi_{0}$ is the potential due to the incident and diffracted waves, while $\phi_{j}(j=1 \sim 6)$ refer to the radiation potentials due to body motions of amplitude $\alpha_{j}$ in six degrees of freedom. Based on the definition of the rigid-body motion modes, the indices 1, 2, 3 refer to surge, sway and heave, while 4, 5, 6 as roll, pitch and yaw, respectively. Each of the velocity potentials satisfies the threedimensional Laplace equation in the fluid domain, which can be written as

$$
\nabla^{2} \phi_{j}+\frac{\partial^{2} \phi_{j}}{\partial z^{2}}=0
$$

where $\nabla^{2}$ is two dimensional Laplacian and is defined as 


$$
\nabla^{2}=\frac{\partial^{2}}{\partial x^{2}}+\frac{\partial^{2}}{\partial y^{2}}=\frac{\partial^{2}}{\partial r^{2}}+\frac{1}{r} \frac{\partial}{\partial r}+\frac{1}{r^{2}} \frac{\partial^{2}}{\partial \theta^{2}}
$$

The linearized free surface boundary condition can be written as

$$
\frac{\partial \phi_{j}}{\partial z}=\frac{\omega^{2}}{g} \phi_{j}, z=0, r<c, r_{1}>a
$$

where $g$ is the acceleration due to gravity. The boundary condition on the ice sheet can be written as (Fox \& Squire, 1994; Meylan \& Squire, 1994)

$$
\left(L \nabla^{4}-m_{i c e} \omega^{2}+\rho g\right) \cdot \frac{\partial \phi_{j}}{\partial z}=\rho \omega^{2} \phi_{j}, \quad z=0, r>c
$$

where $L=E h^{3} /\left[12\left(1-v^{2}\right)\right]$ and $m_{i c e}=\rho_{i c e} h$ are respectively the effective flexural rigidity and the mass per unit area of the ice sheet, $E$ and $v$ are the Young's modulus and Poisson's ratio of the ice, respectively. $\rho$ in the equation is the density of water. On the flat bottom of the fluid $z=-H$, the boundary condition can be written as

$$
\frac{\partial \phi_{j}}{\partial z}=0
$$

The impermeable boundary condition for diffraction problem on the body surface $S_{b}$ or $r_{1}=a$ gives

$$
\frac{\partial \phi_{0}}{\partial n}=-\frac{\partial \phi_{0}}{\partial r_{1}}=0
$$

where $\boldsymbol{n}$ is the normal vector pointing into the body surface. For the translational motion of a bottom-mouthed cylinder, the boundary condition for surge motion can be written as

$$
\frac{\partial \phi_{1}}{\partial n}=-\frac{\partial \phi_{1}}{\partial r_{1}}=-\cos \theta_{1}
$$

For the rotational motion, we follow the case in Drake et al. (1984), in which the cylinder is articulated at the bottom and is allowed to rotate in the $x O z$ plane, or 
the pitch motion is allowed. The corresponding boundary condition can then be written as

$$
\frac{\partial \phi_{5}}{\partial n}=-\frac{\partial \phi_{5}}{\partial r_{1}}=-(H+z) \times \cos \theta_{1}
$$

In the far-field, all the potential components should satisfy the radiation boundary condition below

$$
\frac{\partial \phi}{\partial r}=i \kappa_{0} \phi, r \rightarrow \infty
$$

to ensure that the disturbed wave will propagate outwards, where $\phi$ refers to the radiation potential $\phi_{j}(j=1 \sim 6)$ or the diffracted potential ( $\phi_{0}$ without the incident potential) and $\kappa_{0}$ is the wave number which will be discussed later. It should be noted that the velocity potential corresponding to sway and roll motions can be obtained from the results of the surge and pitch motions of the cylinder in a different location, respectively. As shown in the Figure 1b, for the sway (roll) motion of a cylinder located at $\left(r_{0}, \theta_{0}\right)$, it is the same as the surge (pitch) motion of the cylinder at $\left(r_{0}, \theta_{0} \pm \pi / 2\right)$.

As the body surface and interface between domains $I$ and $I I$ are both circular, this allows us to use variable separation method in the corresponding polar coordinate systems. The incident potential for the incoming wave of amplitude $A$ beneath the ice sheet can be written as

$$
\phi_{i n}=-A \frac{i g}{\omega} \times \frac{\cosh \kappa_{0}(H+z)}{\cosh \kappa_{0} H} \times e^{i \kappa_{0} x}=-A \frac{i g}{\omega} \times \frac{\cosh \kappa_{0}(H+z)}{\cosh \kappa_{0} H} \times e^{i \kappa_{0} r \cos \theta}
$$

Using Eqs. (9.1.44-9.1.45) of Abramowitz \& Stegun (1965), this can be further expanded as 


$$
\phi_{i n}=-A \frac{i g}{\omega} \times \frac{\cosh \kappa_{0}(H+z)}{\cosh \kappa_{0} H} \times \sum_{m=-\infty}^{\infty} e^{i m \pi / 2} \times J_{m}\left(\kappa_{0} r\right) \times e^{i m \theta}
$$

where $J_{m}$ is the Bessel function of the first kind. The expression of the total potential in domain II below the ice sheet can be given as

$$
\phi_{j}^{(I I)}=\delta_{j 0} \times \phi_{i n}+\sum_{n=-2}^{\infty} \sum_{m=-\infty}^{\infty} P_{n m}^{(j)} \times \psi_{n m} \quad \text { for } c \leq r \leq \infty
$$

where $\delta_{i j}$ is the Kronecker delta function,

$$
\psi_{n m}=e^{i m \theta} \times \frac{H_{m}^{(1)}\left(\kappa_{n} r\right)}{H_{m}^{(1)}\left(\kappa_{n} c\right)} \times \frac{\cosh \kappa_{n}(H+z)}{\cosh \kappa_{n} H}
$$

and $H_{m}^{(1)}$ is the Hankel function of the first kind. In Eq. (11), $\kappa_{n}$ are eigenvalues which can be determined by substituting Eq. (10) into (4). This gives

$$
\kappa_{n} \times \tanh \kappa_{n} H=\frac{\rho \omega^{2}}{L \kappa_{n}^{4}+\rho g-m_{i c e} \omega^{2}}, n=-2,-1,0,1,2 \ldots
$$

in which $\kappa_{0}$ is the positive real root, $\kappa_{-2}$ and $\kappa_{-1}$ are the two complex roots with positive imaginary part, and $\kappa_{n}(n=1,2,3 \ldots)$ are the positive pure imaginary roots. The potential written in terms of Eq. (10) satisfies the governing equation and all boundary conditions apart from the continuity condition on the interface. Similarly, the potential of total wave in domain $I$ below open water surface can be written in $\left(r_{1}, \theta_{1}, z\right)$ as

$$
\begin{array}{r}
\phi_{j}^{(I)}\left(r_{1}, \theta_{1}, z\right)=\sum_{n=0}^{\infty} \sum_{m=-\infty}^{\infty} \frac{\left[C_{n m}^{(j)} \times H_{m}^{(2)}\left(k_{n} r_{1}\right)+D_{n m}^{(j)} \times H_{m}^{(1)}\left(k_{n} r_{1}\right)\right] \times e^{i m \theta_{1}} \times H_{m}^{(1)^{\prime}}\left(k_{n} a\right)}{H_{m}^{(2)}\left(k_{n} c\right) \times H_{m}^{(1)^{\prime}}\left(k_{n} a\right)-H_{m}^{(2)^{\prime}}\left(k_{n} a\right) \times H_{m}^{(1)}\left(k_{n} c\right)} \times \frac{\cosh k_{n}(H+z)}{\cosh k_{n} H} \\
, \text { for } r_{1}>a \text { and } r<c
\end{array}
$$

$H_{m}^{(2)}$ is the Hankel function of the second kind and the eigenvalues $k_{n}$ satisfy the following dispersion equation 


$$
k_{n} \times \tanh k_{n} H=\frac{\omega^{2}}{g}, \quad n=0,1,2 \ldots
$$

which can be obtained by substituting Eq. (13) into (3). In Eq. (14), $k_{0}$ is the positive real root, and $k_{n}(n=1,2,3 \ldots)$ are the positive pure imaginary roots. Eq. (13) satisfies Eqs. (2), (3) and (5). The remaining boundary conditions for Eqs. (10) and (13) are that on the body surface and the continuity requirement of the potential and velocity on the interface at $r=c$. To impose the former one, we use Eq. (6). When the orthogonality of the cosine functions with respect to $\theta$ and $\cosh k_{n}(z+H)$ with respect to $z$ are used, we have

$$
\frac{\left[C_{n m}^{(j)} \times H_{m}^{(2) \prime}\left(k_{n} a\right)+D_{n m}^{(j)} \times H_{m}^{(1)^{\prime}}\left(k_{n} a\right)\right] \times H_{m}^{(1)^{\prime}}\left(k_{n} a\right)}{H_{m}^{(2)}\left(k_{n} c\right) \times H_{m}^{(1))^{\prime}}\left(k_{n} a\right)-H_{m}^{(2) \prime}\left(k_{n} a\right) \times H_{m}^{(1)}\left(k_{n} c\right)} \times k_{n}=A_{n m}^{(j)}
$$

where

$$
\begin{gathered}
A_{n m}^{(0)}=0 \\
A_{n m}^{(1)}=\left(\delta_{m 1}+\delta_{m,-1}\right) \times \frac{2 \times \sinh k_{n} H \times \cosh k_{n} H}{\sinh 2 k_{n} H+2 k_{n} H}
\end{gathered}
$$

and

$$
A_{n m}^{(5)}=\left(\delta_{m 1}+\delta_{m,-1}\right) \times 2 \cosh k_{n} H \times \frac{k_{n} H \sinh k_{n} H-\cosh k_{n} H+1}{k_{n} \sinh 2 k_{n} H+2 k_{n}^{2} H}
$$

Thus

$$
D_{n m}^{(j)}=\frac{A_{n m}^{(j)} \times\left[H_{m}^{(2)}\left(k_{n} c\right) \times H_{m}^{(1)^{\prime}}\left(k_{n} a\right)-H_{m}^{(2)^{\prime}}\left(k_{n} a\right) \times H_{m}^{(1)}\left(k_{n} c\right)\right]}{k_{n} \times\left[H_{m}^{(1)^{\prime}}\left(k_{n} a\right)\right]^{2}}-C_{n m}^{(j)} \times \frac{H_{m}^{(2)^{\prime}}\left(k_{n} a\right)}{H_{m}^{(1)^{\prime}}\left(k_{n} a\right)}
$$

Eq. (13) can then be written as 


$$
\phi_{j}^{(I)}\left(r_{1}, \theta_{1}, z\right)=\sum_{n=0}^{\infty} \sum_{m=-\infty}^{\infty}\left(\begin{array}{l}
C_{n m}^{(j)} \times \frac{H_{m}^{(2)}\left(k_{n} r_{1}\right) \times H_{m}^{(1)^{\prime}}\left(k_{n} a\right)-H_{m}^{(2){ }^{\prime}}\left(k_{n} a\right) \times H_{m}^{(1)}\left(k_{n} r_{1}\right)}{H_{m}^{(2)}\left(k_{n} c\right) \times H_{m}^{(1)^{\prime}}\left(k_{n} a\right)-H_{m}^{(2)^{\prime}}\left(k_{n} a\right) \times H_{m}^{(1)}\left(k_{n} c\right)} \\
+\frac{A_{n m}^{(j)}}{k_{n} \times H_{m}^{(1)}\left(k_{n} a\right)} \times H_{m}^{(1)}\left(k_{n} r_{1}\right)
\end{array}\right) \times e^{i m \theta_{1}} \times \frac{\cosh k_{n}(H+z)}{\cosh k_{n} H}
$$

To achieve the continuity requirement at the interface $(r=c)$, Eq. (15) in coordinate system $\left(r_{1}, \theta_{1}\right)$ should be converted to that in $(r, \theta)$. Following Eatock Taylor \& Hung (1985), we may use the Graf's addition theorem for Bessel functions (Abramowitz \& Stegun, 1965)

$$
\mathscr{C}_{m}\left(k_{n} r_{1}\right) \times e^{i m\left(\theta_{1}-\theta\right)}=\sum_{l=-\infty}^{\infty} \mathscr{Q}_{m+l}\left(k_{n} r\right) J_{l}\left(k_{n} r_{0}\right) \times e^{i l\left(\theta-\theta_{0}\right)}, r>r_{0}
$$

where $\mathscr{O}_{m}$ can be any Bessel functions such as $J_{m}, Y_{m}, H_{m}^{(1)}$ and $H_{m}^{(2)}$. Eq. (15) can be transformed to $(r, \theta)$ as

$$
\begin{aligned}
& \phi_{j}^{(I)}(r, \theta, z)= \\
& \sum_{n=0}^{\infty} \sum_{m=-\infty}^{\infty}\left(\begin{array}{c}
C_{n m}^{(j)} \times e^{i m \theta} \times\left(\begin{array}{c}
H_{m}^{(1)^{\prime}}\left(k_{n} a\right) \times \sum_{l=-\infty}^{\infty} H_{m+l}^{(2)}\left(k_{n} r\right) \times J_{l}\left(k_{n} r_{0}\right) \times e^{i l\left(\theta-\theta_{0}\right)} \\
\left.-H_{m}^{(2))^{\prime}}\left(k_{n} a\right) \times \sum_{l=-\infty}^{\infty} H_{m+l}^{(1)}\left(k_{n} r\right) \times J_{l}\left(k_{n} r_{0}\right) \times e^{i l\left(\theta-\theta_{0}\right)}\right)
\end{array}\right) \times \frac{\cosh k_{n}(H+z)}{\cosh k_{n} H} \\
+\frac{H_{m}^{(1)^{\prime}}\left(k_{n} a\right) \times H_{m}^{(2)}\left(k_{n} c\right)-H_{m}^{(2)^{\prime}}\left(k_{n} a\right) \times H_{m}^{(1)}\left(k_{n} c\right)}{k_{n} \times H_{m}^{(1) \prime}\left(k_{n} a\right)} \times e^{i m \theta} \times \sum_{l=-\infty}^{\infty} H_{m+l}^{(1)}\left(k_{n} r\right) \times J_{l}\left(k_{n} r_{0}\right) \times e^{i l\left(\theta-\theta_{0}\right)} \\
j=0,1,5 ; \text { for } r_{1}>a, r>r_{0} \text { and } r<c
\end{array}\right.
\end{aligned}
$$

\section{Solution procedure}

The matching procedure is conducted on the interface surface $r=c$ to obtain the unknown coefficients. For the potential, we may write 


$$
\phi_{j}^{(I)}=\phi_{j}^{(I I)}, \quad r=c
$$

Multiplying both sides with $e^{i m^{\prime} \theta}$ and $\frac{\cosh k_{n^{\prime}}(H+z)}{\cosh k_{n^{\prime}} H}$, integrating with respect to $\theta$ and $z$, and using the orthogonality, we obtain

$$
\begin{aligned}
& \sum_{m=-\infty}^{\infty} C_{n^{\prime} m}^{(j)} \times \frac{H_{-m^{\prime}}^{(2)}\left(k_{n^{\prime}} c\right) \times H_{m}^{(1) '}\left(k_{n^{\prime}} a\right)-H_{m}^{(2))^{\prime}}\left(k_{n^{\prime}} a\right) \times H_{-m^{\prime}}^{(1)}\left(k_{n^{\prime}} c\right)}{H_{m}^{(2)}\left(k_{n^{\prime}} c\right) \times H_{m}^{(1) '}\left(k_{n^{\prime}} a\right)-H_{m}^{(2))^{\prime}}\left(k_{n^{\prime}} a\right) \times H_{m}^{(1)}\left(k_{n^{\prime}} c\right)} \times J_{-m^{\prime}-m}\left(k_{n^{\prime}} r_{0}\right) \times e^{i\left(m+m^{\prime}\right) \theta_{0}} \times \mathrm{X}\left(k_{n^{\prime}}, k_{n^{\prime}}\right) \\
& -\sum_{n=-2}^{\infty} P_{n,-m^{\prime}}^{(j)} \times \mathrm{X}\left(k_{n^{\prime}}, \kappa_{n}\right) \\
& =-\delta_{j 0} \times A \frac{i g}{\omega} \times e^{-i m^{\prime} \pi / 2} \times J_{-m^{\prime}}\left(\kappa_{0} c\right) \times \mathrm{X}\left(k_{n^{\prime}}, \kappa_{0}\right) \\
& -\sum_{m=-\infty}^{\infty} \frac{A_{n^{\prime} m}^{(j)}}{k_{n^{\prime}} \times H_{m}^{(1) \prime}\left(k_{n^{\prime}} a\right)} \times e^{i\left(m+m^{\prime} \theta_{0}\right.} \times H_{-m^{\prime}}^{(1)}\left(k_{n^{\prime}} c\right) \times J_{-m^{\prime}-m}\left(k_{n^{\prime}}, r_{0}\right) \times \mathrm{X}\left(k_{n^{\prime}}, k_{n^{\prime}}\right)
\end{aligned}
$$

where $\mathrm{X}\left(x_{1}, x_{2}\right)=\frac{x_{1} \tanh x_{1} H-x_{2} \tanh x_{2} H}{x_{1}^{2}-x_{2}^{2}}$, for $x_{1} \neq x_{2}$, and by taking the limit we can obtain $\mathrm{X}(x, x)=\frac{1}{4 \cosh ^{2} x H} \times\left(\frac{\sinh 2 x H}{x}+2 H\right)$ when $x_{1}=x_{2}=x$.

To impose the continuity condition of the normal velocity, we can use the Green's second identity in domain II. For two harmonic functions $\varphi$ and $\psi$, we have,

$$
\oiint_{S}\left[\varphi \frac{\partial \psi}{\partial n}-\psi \frac{\partial \varphi}{\partial n}\right] d S=0
$$

where $S$ is the boundary of domain $I I$. Let $\varphi=\phi_{j}^{(I I)}-\delta_{j 0} \times \phi_{i n}$ and $\psi=\psi_{n^{\prime} m^{\prime}}$ from Eq. (11). The integration over the bottom of the liquid and the surface at $r \rightarrow \infty$ will disappear when the corresponding boundary condition is used. The integration in Eq. (20) is then over the interface $S_{0}$ at $r=c$ and the ice sheet $S_{I}$ only. We have 


$$
\begin{aligned}
& \iint_{S_{0}}\left[-\left(\phi_{j}^{(I)}-\delta_{j 0} \times \phi_{i n}\right) \times \frac{\partial \psi_{n^{\prime} m^{\prime}}}{\partial r}+\psi_{n^{\prime} m^{\prime}} \times \frac{\partial}{\partial r}\left(\phi_{j}^{(I I)}-\delta_{j 0} \times \phi_{i n}\right)\right] d S \\
& +\iint_{S_{I}}\left[\left(\phi_{j}^{(I I)}-\delta_{j 0} \times \phi_{i n}\right) \times \frac{\partial \psi_{n^{\prime} m^{\prime}}}{\partial z}-\psi_{n^{\prime} m^{\prime}} \times \frac{\partial\left(\phi_{j}^{(I I)}-\delta_{j 0} \times \phi_{i n}\right)}{\partial z}\right] d S=0
\end{aligned}
$$

For the second term of Eq. (21), we use the boundary condition in Eq. (4)

$$
\phi_{j}^{(I I)}-\delta_{j 0} \times \phi_{i n}=\frac{L}{\rho \omega^{2}} \nabla^{4} f+\frac{\rho g-m_{i c} \omega^{2}}{\rho \omega^{2}} f \quad \text { for } z=0
$$

where $f=\frac{\partial}{\partial z}\left(\phi_{j}^{(I I)}-\delta_{j 0} \times \phi_{i n}\right)$. Similarly, $\psi_{n^{\prime} m^{\prime}}$ also satisfies Eq. (4), or

$$
\psi_{n^{\prime} m^{\prime}}=\frac{L}{\rho \omega^{2}} \nabla^{4} q+\frac{\rho g-m_{i c e} \omega^{2}}{\rho \omega^{2}} q \text { for } z=0
$$

where $q=\frac{\partial \psi_{n^{\prime} m^{\prime}}}{\partial z}$. Substituting Eqs. (22) and (23) into the second term of Eq. (21) and using the Gauss theorem on $S_{I}$, the surface integral becomes line integral

$$
\begin{aligned}
& \iint\left[\left(\frac{L}{\rho \omega^{2}} \nabla^{4} f+\frac{\rho g-m_{i c} \omega^{2}}{\rho \omega^{2}} f\right) q-\left(\frac{L}{\rho \omega^{2}} \nabla^{4} q+\frac{\rho g-m_{i c e} \omega^{2}}{\rho \omega^{2}} q\right) f\right] d S \\
& =\frac{L}{\rho \omega^{2}} \times \iint_{S_{I}}\left[\left(\nabla^{4} f\right) q-\left(\nabla^{4} q\right) f\right] d S \\
& =-\frac{L}{\rho \omega^{2}} \times \oint_{C_{1}}\left(q \frac{\partial}{\partial n} \nabla^{2} f-\frac{\partial q}{\partial n} \nabla^{2} f-f \frac{\partial}{\partial n} \nabla^{2} q+\frac{\partial f}{\partial n} \nabla^{2} q\right) d s
\end{aligned}
$$

where $\boldsymbol{n}$ is the normal vector of the ice edge pointing inward the domain II. The line integral at infinite $C_{\infty}$ in Eq. (24) has disappeared after the radiation condition in Eq. (7) is used. The line integral along the edge of the ice sheet $C_{1}$ can be further treated through applying the edge conditions. The detailed procedure can be found in Appendix A. Based on Eq. (21), the equations for the unknown coefficients can be finally given as 


$$
\begin{aligned}
& \sum_{n=-2}^{\infty} P_{n,-m^{\prime}}^{(j)} \times\left\{\begin{array}{l}
-\kappa_{n^{\prime}} \times \frac{H_{m^{\prime}}^{(1)}\left(\kappa_{n^{\prime}} c\right)}{H_{m^{\prime}}^{(1)}\left(\kappa_{n^{\prime}} c\right)} \times \mathrm{X}\left(\kappa_{n}, \kappa_{n^{\prime}}\right) \\
-\frac{L}{\rho \omega^{2}} \times \tanh \kappa_{n} H \times \tanh \kappa_{n^{\prime}} H \times \zeta_{1}\left(n, n^{\prime}, m^{\prime}\right)
\end{array}\right\} \\
& +\sum_{n=0}^{\infty} \sum_{m=-\infty}^{\infty} C_{n m}^{(j)} \times \frac{H_{-m^{\prime}}^{(2)}\left(k_{n} c\right) \times H_{m}^{(1))^{\prime}}\left(k_{n} a\right)-H_{m}^{(2) \prime}\left(k_{n} a\right) \times H_{-m^{\prime}}^{(1)}\left(k_{n} c\right)}{H_{m}^{(2)}\left(k_{n} c\right) \times H_{m}^{(1))^{\prime}}\left(k_{n} a\right)-H_{m}^{(2) \prime}\left(k_{n} a\right) \times H_{m}^{(1)}\left(k_{n} c\right)} \times k_{n} \times J_{-m-m^{\prime}}\left(k_{n} r_{0}\right) \times e^{i\left(m+m^{\prime}\right) \theta_{0}} \times \mathrm{X}\left(k_{n}, \kappa_{n^{\prime}}\right) \\
& =-\sum_{n=0}^{\infty} \sum_{m=-\infty}^{\infty}\left(\frac{A_{n m}^{(j)}}{k_{n} \times H_{m}^{(1) \prime}\left(k_{n} a\right)} \times k_{n} \times H_{-m^{\prime}}^{(1)}\left(k_{n} c\right) \times J_{-m-m^{\prime}}\left(k_{n} r_{0}\right) \times e^{i\left(m+m^{\prime}\right) \theta_{0}}\right) \times \mathrm{X}\left(k_{n}, \kappa_{n^{\prime}}\right) \\
& -\delta_{j 0} \times\left\{\begin{array}{l}
A \frac{i g}{\omega} \times e^{-i m^{\prime} \pi / 2} \times \kappa_{0} \times J_{-m^{\prime}}^{\prime}\left(\kappa_{0} c\right) \times \mathrm{X}\left(\kappa_{0}, \kappa_{n^{\prime}}\right) \\
+\frac{L}{\rho \omega^{2}} \times A \frac{i g}{\omega} \times \kappa_{0} \times \tanh \kappa_{0} H \times e^{-i m^{\prime} \pi / 2} \times \kappa_{n^{\prime}} \times \tanh \kappa_{n^{\prime}} H \times \zeta_{2}\left(n^{\prime}, m^{\prime}\right)
\end{array}\right\}
\end{aligned}
$$

where

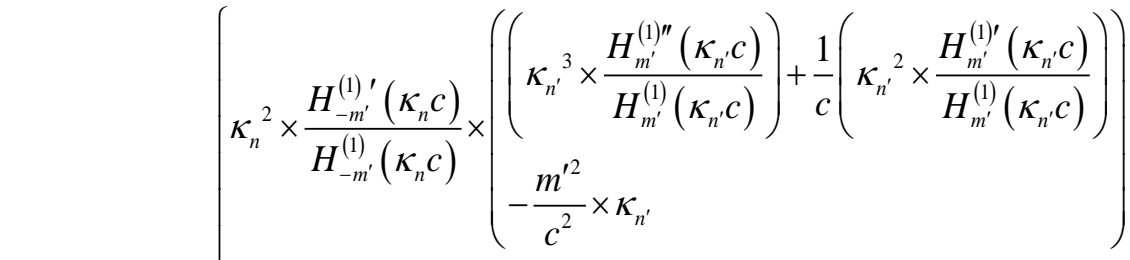

$$
\begin{aligned}
& \zeta_{1}\left(n, n^{\prime}, m^{\prime}\right)=\left\{-\kappa_{n} \times\left(\left(\kappa_{n^{\prime}}{ }^{4} \times \frac{H_{m^{\prime}}^{(1) \prime^{\prime \prime}}\left(\kappa_{n^{\prime}} c\right)}{H_{m^{\prime}}^{(1)}\left(\kappa_{n^{\prime}} c\right)}\right)-\frac{1+m^{\prime 2}}{c^{2}}\left(\kappa_{n^{\prime}}{ }^{2} \times \frac{H_{m^{\prime}}^{(1) '}\left(\kappa_{n^{\prime}} c\right)}{H_{m^{\prime}}^{(1)}\left(\kappa_{n^{\prime}} c\right)}\right)+\frac{1}{c}\left(\kappa_{n^{\prime}}{ }^{3} \times \frac{H_{m^{\prime}}^{(1) \prime}\left(\kappa_{n^{\prime}} c\right)}{H_{m^{\prime}}^{(1)}\left(\kappa_{n^{\prime}} c\right)}\right)+\frac{2 m^{\prime 2}}{c^{3}} \times \kappa_{n^{\prime}}\right)\right. \\
& +\frac{(1-v)}{c^{2}} \times\left(\kappa_{n} \times \frac{H_{-m^{\prime}}^{(1)}\left(\kappa_{n} c\right)}{H_{-m^{\prime}}^{(1)}\left(\kappa_{n} c\right)}-\frac{1}{c}\right) \times m^{\prime 2} \times \kappa_{n} \times \kappa_{n^{\prime}} \\
& -\frac{(1-v)}{c} \times\left(\kappa_{n} \times \frac{H_{-m^{\prime}}^{(1)}\left(\kappa_{n} c\right)}{H_{-m^{\prime}}^{(1)}\left(\kappa_{n} c\right)}-\frac{m^{\prime 2}}{c}\right) \times \kappa_{n} \times \kappa_{n^{\prime}}{ }^{2} \times \frac{H_{m^{\prime}}^{(1)}\left(\kappa_{n^{\prime}} c\right)}{H_{m^{\prime}}^{(1)}\left(\kappa_{n^{\prime}} c\right)}
\end{aligned}
$$

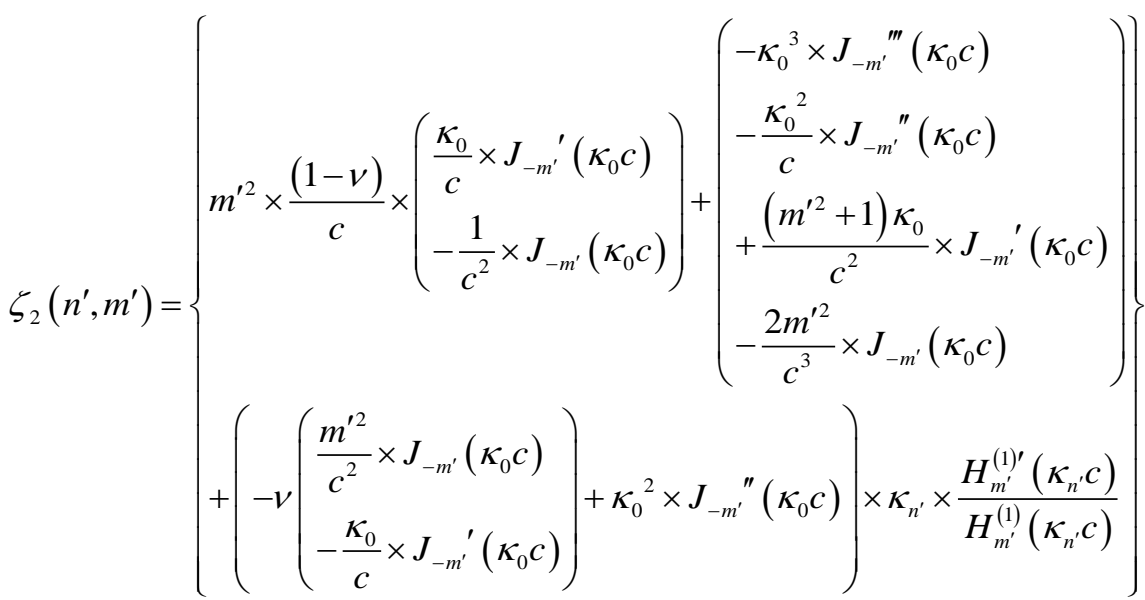


Then, Eqs. (19) and (25) compose a system of linear equations for the unknown coefficients $C_{n m}^{(j)}$ and $P_{n m}^{(j)}$. The infinite series need to be truncated for the practical calculation. Specifically, $n$ and $n^{\prime}$ range from 0 to $N-1$ for $k_{n}\left(k_{n^{\prime}}\right)$ and from -2 to $N-3$ for $\kappa_{n}\left(\kappa_{n^{\prime}}\right), m$ and $m^{\prime}$ range from $-M$ to $M . N$ and $M$ should be chosen large enough to ensure the convergence and accuracy of the results. Finally, we have the system of linear equations as

$$
\left[\begin{array}{ll}
\mathscr{F}^{(11)} & \mathscr{H}^{(12)} \\
\mathscr{F}^{(21)} & \mathscr{S}^{(22)}
\end{array}\right] \cdot \boldsymbol{X}=\left\{\begin{array}{l}
\mathscr{B}^{(j, 1)} \\
\mathscr{B}^{(j, 2)}
\end{array}\right\}
$$

where $\boldsymbol{X}$ is a column containing $2 N \times(2 M+1)$ elements and $X_{k}$ is denoted as $X_{k}= \begin{cases}C_{n m}^{(j)}, & k=(2 M+1) \times n+(M+1-m), 0 \leq n \leq N-1,-M \leq m \leq M \\ P_{n m}^{(j)}, & k=(2 M+1) \times N+(2 M+1) \times(n+2)+(M+1-m),-2 \leq n \leq N-3,-M \leq m \leq M\end{cases}$ while $\mathscr{H}^{(11)}, \mathscr{H}^{(12)}, \mathscr{F}^{(21)}, \mathscr{H}^{(22)}$ are square matrices of order $N \times(2 M+1)$ and their coefficients can be obtained respectively as

$$
\begin{aligned}
& \mathscr{F}_{i k}^{(11)}=\left\{\begin{array}{l}
\frac{H_{-m^{\prime}}^{(2)}\left(k_{n^{\prime}} c\right) \times H_{m}^{(1)^{\prime}}\left(k_{n^{\prime}} a\right)-H_{m}^{(2) '}\left(k_{n^{\prime}} a\right) \times H_{-m^{\prime}}^{(1)}\left(k_{n^{\prime}} c\right)}{H_{m}^{(2)}\left(k_{n^{\prime}} c\right) \times H_{m}^{(1)^{\prime}}\left(k_{n^{\prime}} a\right)-H_{m}^{(2) '}\left(k_{n^{\prime}} a\right) \times H_{m}^{(1)}\left(k_{n^{\prime}} c\right)} \times J_{-m^{\prime}-m}\left(k_{n^{\prime}} r_{0}\right) \times e^{i\left(m+m^{\prime}\right) \theta_{0}} \times \mathrm{X}\left(k_{n^{\prime}}, k_{n^{\prime}}\right), n=n^{\prime} \\
0, n \neq n^{\prime}
\end{array}\right. \\
& i=(2 M+1) \times n^{\prime}+\left(M+1+m^{\prime}\right), k=(2 M+1) \times n+(M+1-m), 0 \leq n, n^{\prime} \leq N-1,-M \leq m, m^{\prime} \leq M \\
& \mathscr{S}_{i k}^{(12)}=\left\{\begin{array}{l}
-\mathrm{X}\left(k_{n^{\prime}}, \kappa_{n}\right), m=-m^{\prime} \\
0, m \neq-m^{\prime}
\end{array}\right. \\
& i=(2 M+1) \times n^{\prime}+\left(M+1+m^{\prime}\right), k=(2 M+1) \times(n+2)+(M+1-m),-2 \leq n \leq N-3,0 \leq n^{\prime} \leq N-1,-M \leq m, m^{\prime} \leq M \\
& \mathscr{F}_{i k}^{(21)}=\frac{H_{-m^{\prime}}^{(2)}\left(k_{n} c\right) \times H_{m}^{(1)^{\prime}}\left(k_{n} a\right)-H_{m}^{(2) '}\left(k_{n} a\right) \times H_{-m \prime^{\prime}}^{(1)}{ }^{\prime}\left(k_{n} c\right)}{H_{m}^{(2)}\left(k_{n} c\right) \times H_{m}^{(1)^{\prime}}\left(k_{n} a\right)-H_{m}^{(2)}\left(k_{n} a\right) \times H_{m}^{(1)}\left(k_{n} c\right)} \times k_{n} \times J_{-m-m^{\prime}}\left(k_{n} r_{0}\right) \times e^{i\left(m+m^{\prime} \theta_{0}\right.} \times \mathrm{X}\left(k_{n}, \kappa_{n^{\prime}}\right) \\
& i=(2 M+1) \times\left(n^{\prime}+2\right)+\left(M+1+m^{\prime}\right), k=(2 M+1) \times n+(M+1-m), 0 \leq n \leq N-1,-2 \leq n^{\prime} \leq N-3,-M \leq m, m^{\prime} \leq M \\
& \mathscr{F}_{i k}^{(22)}=\left\{\begin{array}{l}
-\kappa_{n^{\prime}} \times \frac{H_{m^{\prime}}^{(1)}\left(\kappa_{n^{\prime}} c\right)}{H_{m^{\prime}}^{(1)}\left(\kappa_{n^{\prime}} c\right)} \times \mathrm{X}\left(\kappa_{n}, \kappa_{n^{\prime}}\right)-\frac{L}{\rho \omega^{2}} \times \tanh \kappa_{n} H \times \tanh \kappa_{n^{\prime}} H \times \zeta_{1}\left(n, n^{\prime}, m^{\prime}\right), m=-m^{\prime} \\
0, m \neq-m^{\prime}
\end{array}\right. \\
& i=(2 M+1) \times\left(n^{\prime}+2\right)+\left(M+1+m^{\prime}\right), k=(2 M+1) \times(n+2)+(M+1-m),-2 \leq n, n^{\prime} \leq N-3,-M \leq m, m^{\prime} \leq M
\end{aligned}
$$

and 


$$
\begin{aligned}
& \mathscr{O}_{i}^{(j, 1)}=-\delta_{j 0} \times A \frac{i g}{\omega} \times e^{-i m^{\prime} \pi / 2} \times J_{-m^{\prime}}\left(\kappa_{0} c\right) \times \mathrm{X}\left(k_{n^{\prime}}, \kappa_{0}\right) \\
& -\sum_{m=-\infty}^{\infty} \frac{A_{n^{\prime} m}^{(j)}}{k_{n^{\prime}} \times H_{m}^{(1)^{\prime}}\left(k_{n^{\prime}} a\right)} \times e^{i\left(m+m^{\prime}\right) \theta_{0}} \times H_{-m^{\prime}}^{(1)}\left(k_{n^{\prime}} c\right) \times J_{-m^{\prime}-m}\left(k_{n^{\prime}}, r_{0}\right) \times \mathrm{X}\left(k_{n^{\prime}}, k_{n^{\prime}}\right) \\
& i=(2 M+1) \times n^{\prime}+\left(M+1+m^{\prime}\right), 0 \leq n^{\prime} \leq N-1,-M \leq m^{\prime} \leq M \\
& \mathscr{O}_{i}^{(j, 2)}=-\sum_{n=0}^{\infty} \sum_{m=-\infty}^{\infty}\left(\frac{A_{n m}^{(j)}}{k_{n} \times H_{m}^{(1)^{\prime}}\left(k_{n} a\right)} \times k_{n} \times H_{-m^{\prime}}^{(1)}{ }^{\prime}\left(k_{n} c\right) \times J_{-m-m^{\prime}}\left(k_{n} r_{0}\right) \times e^{i\left(m+m^{\prime}\right) \theta_{0}}\right) \times \mathrm{X}\left(k_{n}, \kappa_{n^{\prime}}\right) \\
& -\delta_{j 0} \times\left\{\begin{array}{l}
A \frac{i g}{\omega} \times e^{-i m^{\prime} \pi / 2} \times \kappa_{0} \times J_{-m^{\prime}}{ }^{\prime}\left(\kappa_{0} c\right) \times \mathrm{X}\left(\kappa_{0}, \kappa_{n^{\prime}}\right) \\
+\frac{L}{\rho \omega^{2}} \times A \frac{i g}{\omega} \times \kappa_{0} \times \tanh \kappa_{0} H \times e^{-i m^{\prime} \pi / 2} \times \kappa_{n^{\prime}} \times \tanh \kappa_{n^{\prime}} H \times \zeta_{2}\left(n^{\prime}, m^{\prime}\right)
\end{array}\right\} \\
& i=(2 M+1) \times\left(n^{\prime}+2\right)+\left(M+1+m^{\prime}\right),-2 \leq n^{\prime} \leq N-3,-M \leq m^{\prime} \leq M
\end{aligned}
$$

\section{Wave loads on body}

The existence of ice sheets can make the flow field in the polynya much more complex. It will in turn have major effect on the hydrodynamic forces on the vertical cylinder. The force can be obtained by integrating the hydrodynamic pressure over the body surface. For the linear problem, the total force can be decomposed as the hydrodynamic coefficients and wave exciting forces. The former is due to wave radiation potentials and can be written as

$$
\begin{aligned}
\tau_{i j}=\mu_{i j}+\frac{i \lambda_{i j}}{\omega}=\rho \times \iint_{S_{b}} \phi_{i} n_{j} d S \\
=-a \pi \rho \times \sum_{n=0}^{\infty}\left(\left(C_{n,-1}^{(i)}+C_{n 1}^{(i)}\right) \times \frac{H_{1}^{(1)^{\prime}}\left(k_{n} a\right) \times H_{1}^{(2)}\left(k_{n} a\right)-H_{1}^{(2)}\left(k_{n} a\right) \times H_{1}^{(1)}\left(k_{n} a\right)}{H_{1}^{(1)^{\prime}}\left(k_{n} a\right) \times H_{1}^{(2)}\left(k_{n} c\right)-H_{1}^{(2)}\left(k_{n} a\right) \times H_{1}^{(1)}\left(k_{n} c\right)}+\left(A_{n,-1}^{(i)}+A_{n 1}^{(i)}\right) \times \frac{H_{1}^{(1)}\left(k_{n} a\right)}{k_{n} \times H_{1}^{(1)^{\prime}}\left(k_{n} a\right)}\right) \\
\quad \times\left(\delta_{j 1} \times \frac{\tanh k_{n} H}{k_{n}}+\delta_{j 5} \times \frac{k_{n} H \sinh k_{n} H-\cosh k_{n} H+1}{k_{n}{ }^{2} \cosh k_{n} H}\right),(i, j=1,5)
\end{aligned}
$$

where $\mu_{i j}$ and $\lambda_{i j}$ are added mass and damping coefficients, respectively. The subscript $i j$ means the coefficient in the $i^{\text {th }}$ mode due to the motion in the $j^{\text {th }}$ mode. Similarly, the wave exciting force on the cylinder can be written as 


$$
\begin{aligned}
& F_{j}^{D}=i \omega \rho \iint_{S_{b}} \phi_{0} n_{j} d S \\
& =-i \omega \rho a \pi \times \sum_{n=0}^{\infty}\left(\left(C_{n,-1}^{(0)}+C_{n 1}^{(0)}\right) \times \frac{H_{1}^{(1)^{\prime}}\left(k_{n} a\right) \times H_{1}^{(2)}\left(k_{n} a\right)-H_{1}^{(2) '}\left(k_{n} a\right) \times H_{1}^{(1)}\left(k_{n} a\right)}{H_{1}^{(1)^{\prime}}\left(k_{n} a\right) \times H_{1}^{(2)}\left(k_{n} c\right)-H_{1}^{(2)}\left(k_{n} a\right) \times H_{1}^{(1)}\left(k_{n} c\right)}\right) \\
& \times\left(\delta_{j 1} \times \frac{\tanh k_{n} H}{k_{n}}+\delta_{j 5} \times \frac{k_{n} H \sinh k_{n} H-\cosh k_{n} H+1}{k_{n}^{2} \cosh k_{n} H}\right),(j=1,5)
\end{aligned}
$$

By using the Green's second identity, the damping coefficient and the exciting force $F_{j}^{D}$ can also be obtained by a far-field method, as shown in Appendixes B and $\mathrm{C}$.

\section{Results and discussion}

The dimensional parameters used for the calculation are:

$$
E=5 \mathrm{Gpa}, \nu=0.3, \rho_{\text {ice }}=922.5 \mathrm{~kg} \mathrm{~m}^{-3}, \rho=1025 \mathrm{~kg} \mathrm{~m}^{-3}, g=9.8 \mathrm{~m} / \mathrm{s}^{2} .
$$

All the variables in our computation can be non-dimensionalized based on the basic parameters, namely, the acceleration due to gravity $g$, the density of water $\rho$ as well as a characteristic length scale. Three cases will be considered below, which are a cylindrical polynya without cylinder, a cylinder located at the centre of polynya and a cylinder located off centre.

(a) A cylindrical polynya

When the vertical cylinder is removed from the open water, there will be only diffraction problem with $A_{n m}^{(0)}=0$. The radius of the polynya $c$ is set as the characteristic length. When $a=0$, the term in Eq. (15) can be simplified using

$$
\frac{H_{m}^{(2)}\left(k_{n} r_{1}\right) \times H_{m}^{(1) '}\left(k_{n} a\right)-H_{m}^{(2) '}\left(k_{n} a\right) \times H_{m}^{(1)}\left(k_{n} r_{1}\right)}{H_{m}^{(2)}\left(k_{n} c\right) \times H_{m}^{(1) '}\left(k_{n} a\right)-H_{m}^{(2) '}\left(k_{n} a\right) \times H_{m}^{(1)}\left(k_{n} c\right)}=\frac{J_{m}\left(k_{n} r_{1}\right)}{J_{m}\left(k_{n} c\right)}
$$


Thus, the wave elevation in the open water can be calculated by the following equation

$$
\eta(r, \theta)=\left.\frac{i \omega}{g} \phi_{0}^{(I)}\right|_{z=0}=\frac{i \omega}{g} \times \sum_{n=0}^{\infty} \sum_{m=-\infty}^{\infty} C_{n m}^{(0)} \times \frac{J_{m}\left(k_{n} r\right)}{J_{m}\left(k_{n} c\right)} \times e^{i m \theta}
$$

When $\omega=0$, Eqs. (3) and (4) will reduce to $\partial \phi_{j} / \partial z=0$ and both surfaces of open water and ice sheet become a rigid lid, and thus the elevation will be equal to zero as can be seen from Eq. (29).

Convergence study is conducted by comparing the results for the wave elevation amplitude along the $x$-axis line within the open water area $(-c \leq x \leq c, y=0, z=0)$. Four cases of incident wave with different wavelength are chosen, namely, $\frac{c}{\lambda}=\frac{1}{2 \pi}, \frac{1}{\pi}, \frac{3}{2 \pi}, \frac{2}{\pi}$, which correspond to wavenumbers $\kappa_{0} c=1,2,3,4$, to cover both long and short waves. We vary $\mathrm{N}$ and $\mathrm{M}$ by selecting $(\mathrm{N}, \mathrm{M})=(50,10),(50,20)$ and $(100,10)$ in the simulation. Figure 2 shows a good agreement among these results from different $\mathrm{N}$ and $\mathrm{M}$ for all the cases of different wavelengths. This means that convergence has been achieved, and $\mathrm{N}=50$ and $\mathrm{M}=10$ will be used in the following calculations.
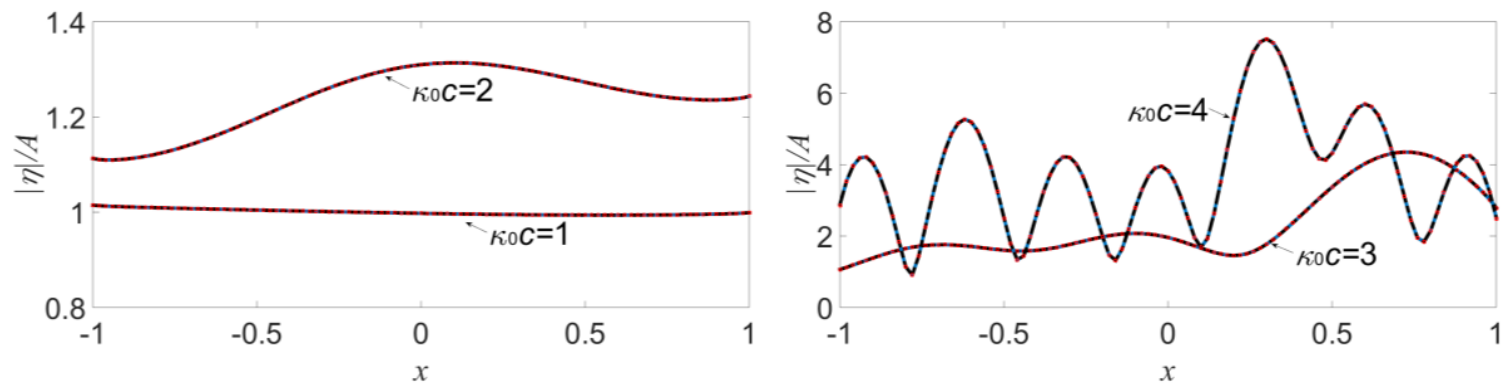

Figure 2. Convergence with respect to $\mathrm{N}$ and $\mathrm{M}$. Solid lines: $\mathrm{N}=50, \mathrm{M}=10$; dashed-dotted lines: $\mathrm{N}=50, \mathrm{M}=20$; squares: $\mathrm{N}=100, \mathrm{M}=10 .\left(c=1, H=2, m_{i c e}=0.036, L=0.05835\right)$ 
Figures $3 a-3 d$ show the free surface profiles at different wavenumbers for the entire inner domain $(r \leq c)$. As the wave inside the polynya is only due to the diffraction of the ice edge, the symmetry of the flow field about the $y=0$ plane is visible from the results. The slight distortion is due to the viewing direction in the 3D. In Figure 3(a), we can see that at small frequency the wave amplitude within the polynya varies very little and in fact it is virtually entirely due to the incoming wave and as a result it is around 1. We notice that when there is no body in the polynya, wave diffraction is entirely due to the difference in the boundary conditions on the free surface $r<c$ and on the ice sheet $r>c$. When $\omega \rightarrow 0$, the leading terms in Eqs. (3) and (4) will tend to the same. This means that wave diffraction will tend to zero as reflected in Figure 3a. When $\kappa_{0} c$ increases, Eqs. (3) and (4) begin to depart from each other. When the incident wave encounters the ice edge, the wave will be significantly altered. The spatial variation of the amplitude becomes faster and the number of peaks and troughs increases as $\kappa_{0} c$ increases. Together with Figure 2, we can also see that there is a region close to the left edge of the ice, where the wave amplitude is quite small, indicating the free surface has very little movement. In fact, as $\kappa_{0} c$ increases, on the $x$ axis, this point further moves to the left and the amplitude is virtually zero, as shown in Figure 2.

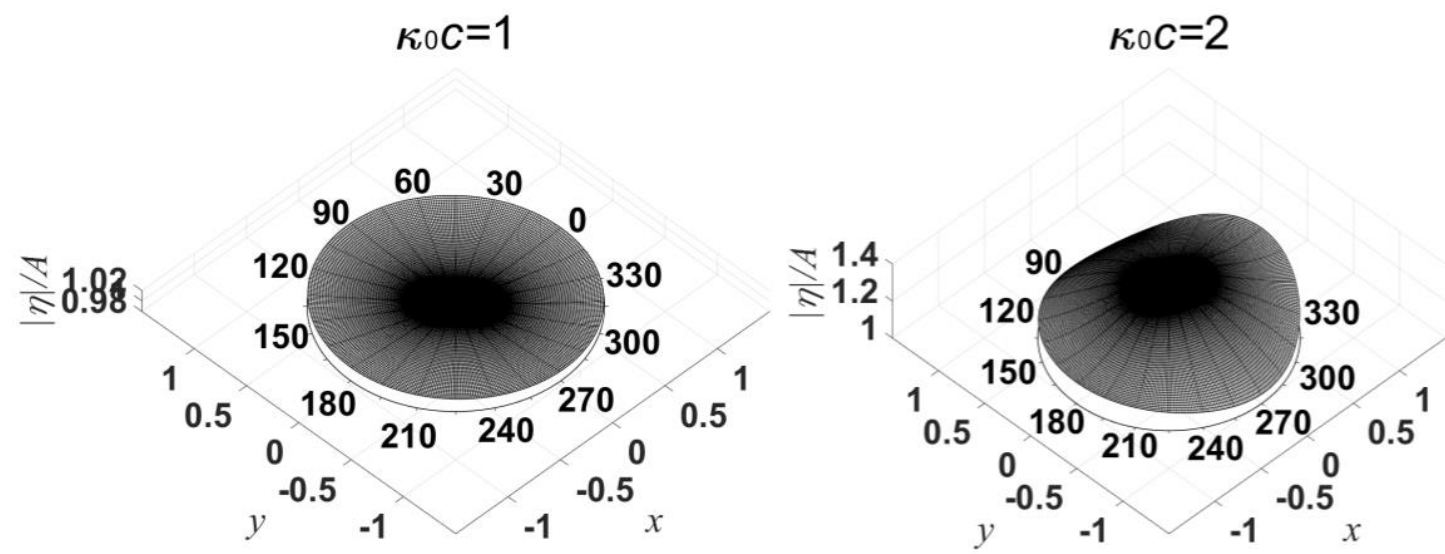




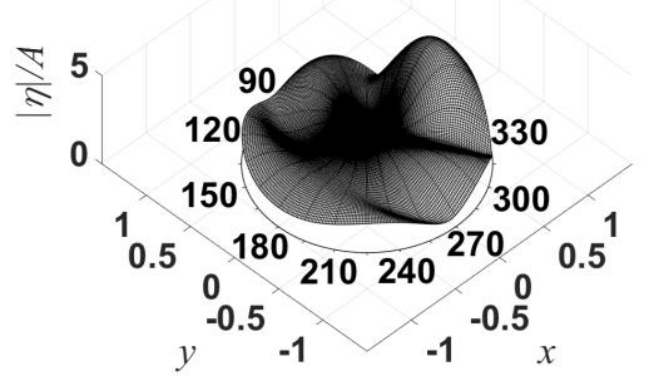

(c)

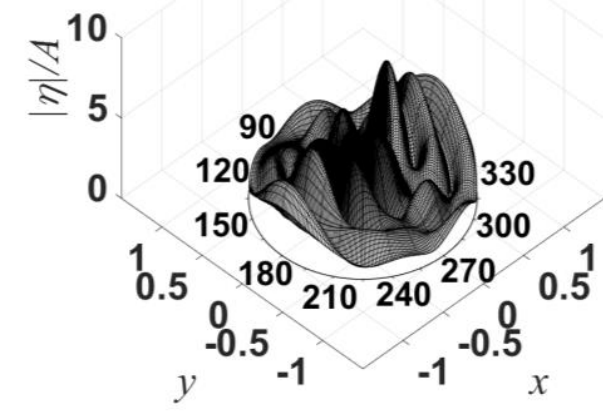

(d)

Figure 3. Wave amplitude patterns in polynya at different wave frequencies $(c=1, H=2$, $m_{i c e}=0.018, L=0.00729$ ). (a) $\kappa_{0} c=1$, (b) $\kappa_{0} c=2$, (c) $\kappa_{0} c=3$, (d) $\kappa_{0} c=4$.

(b) A centrally located cylinder

We now consider a cylinder located at the centre of the polynya, or $r_{0}=0$. Its radius $a$ is used as the characteristic length. As $J_{l}(0)=\delta_{l 0}, r_{1}=r, \theta_{1}=\theta$, Eq. (17) will reduce to Eq. (15). The hydrodynamic coefficients and the wave exciting forces on the cylinder can be obtained by Eqs. (27) and (28), respectively. For the damping coefficients and the exciting forces, the results obtained by far field equations, or Eqs. (B8) and (C3), are used for comparison and excellent agreement has been found.
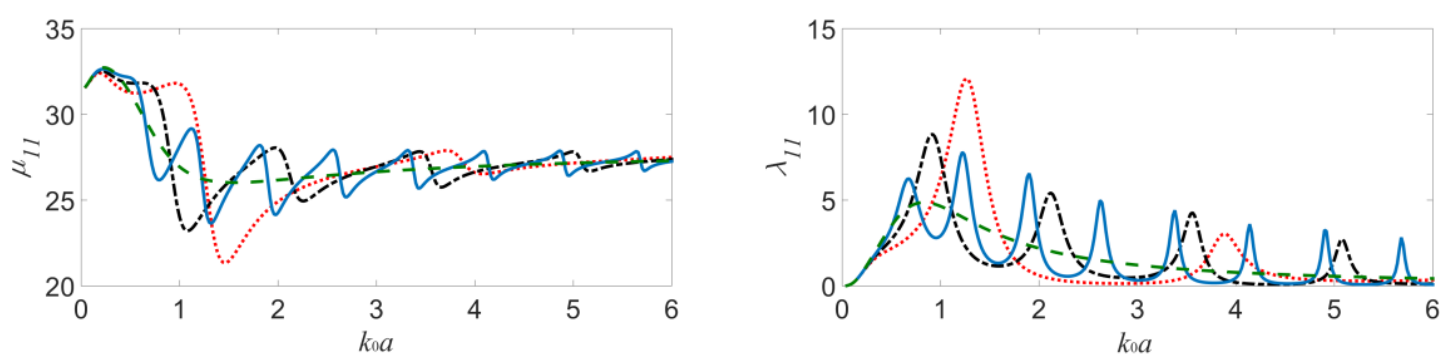

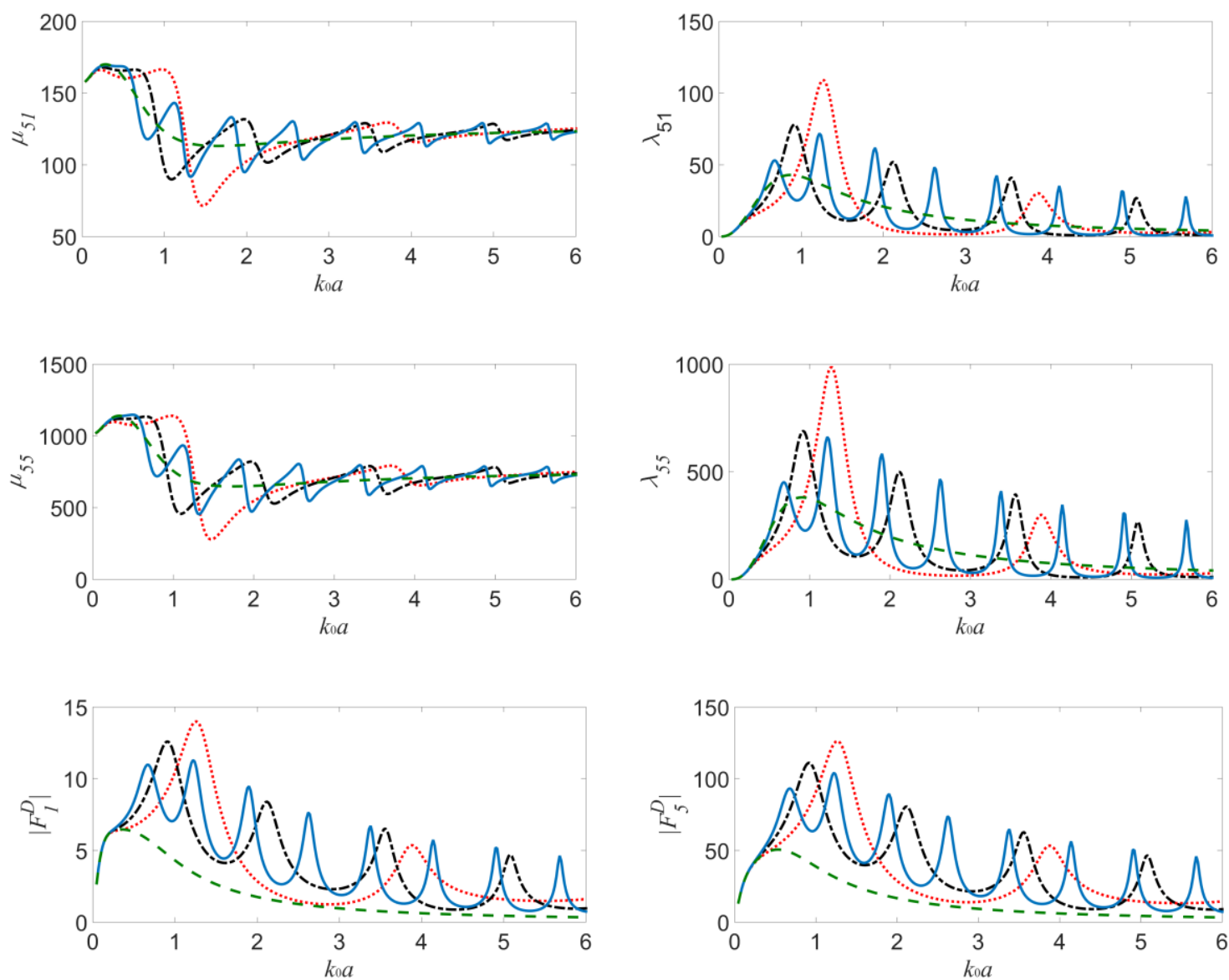

Figure 4. Hydrodynamic coefficients and wave exciting forces against $k_{0} a$ at different $c$. $(a=$ 1, $\left.h=0.1, H=10, m_{i c e}=0.09, L=4.5582\right)$. Dotted lines: $c=2$; dashed-dotted lines: $c=3$; solid lines: $c=5$; dashed lines: ice free.

Results against $k_{0} a$ at different $c$ are given in Figure 4, together with those from the open water. The results show the typical oscillatory behaviour in the polynya. As $c$ increases, the results become more oscillatory. This is against intuition that as $c$ increases, the result might tend to that without ice sheet or in completely open water. When the value $c$ is set to be infinite, the problem will become the classic full free flow problem. However, when $c$ tends to be infinite the problem will not tend to that of the free surface problem, which is consistent with that observed in the two-dimensional problem ( $\mathrm{Li}$ et al 2017). In fact, at any given $c$, no matter how large it is, the wave number $\kappa_{0}$ below the ice sheet will remain the same, and will be different from the wave number $k_{0}$. As a result, the radiation condition in Eq. (7) is always different from that in the open water problem. Thus, the results 
in the polynya will be also different from those in the open water, no matter how large $c$ is.

We may investigate further the oscillatory behaviour of the results shown in Figure 4 by considering the hydrodynamic coefficients. The several peaks shown in our results are due to the oscillatory behaviour of the parameters $C_{n m}$ terms corresponding to the waves reflected by the ice edge. As the terms behind the $C_{n m}$ terms in Eq. (27) is same with that in Eq. (28), the oscillatory of the exciting forces can be also accounted for in a similar way. From Eq. (27), we notice that $A_{n m}$ term is that due to open water and $C_{n m}$ term is due to the ice sheet. When the body oscillates, the generated wave will propagate outwards. Without ice sheet, the wave will propagate to infinity. There will be only $A_{m n}$ which usually does not give the oscillation seen in Figure 4. When there is an ice sheet, the radiated wave be reflected as well as transmitted. When $c$ is much larger than $a$ and wavelength, the evanescent wave with $n>0$ will diminish and only the propagating wave with $n=0$ is significant at the ice edge, which is proportional to $H_{m}^{(1)}\left(k_{0} c\right)$. It can then be expected the solution of $C_{n m}$ and $P_{n m}$ will depend on $c$ in the form $H_{m}^{(1)}\left(k_{0} c\right)$. As the first approximation, $P_{n m}$ may be taken zero, implying the potential transmitted into that the region below the ice sheet is zero. By using the asymptotic expansion of the Hankel functions at large argument $k_{0} c$, we have the $C_{0, \pm 1}$ term in Eq. (27) is proportional to $e^{2 i k_{0} c} /\left(\alpha_{1}+\alpha_{2} \times e^{2 i k_{0} c}\right)$, where

$$
\alpha_{1}=-i \times \frac{H_{1}^{(1)^{\prime}}\left(k_{0} a\right)}{H_{1}^{(1)^{\prime}}\left(k_{0} a\right) \times H_{1}^{(2)}\left(k_{0} a\right)-H_{1}^{(2) \prime^{\prime}}\left(k_{0} a\right) \times H_{1}^{(1)}\left(k_{0} a\right)}=-\frac{H_{1}^{(1)^{\prime}}\left(k_{0} a\right)}{4} \times \pi k_{0} a
$$

and

$$
\alpha_{2}=-\frac{H_{1}^{(2)^{\prime}}\left(k_{0} a\right)}{H_{1}^{(1)^{\prime}}\left(k_{0} a\right) \times H_{1}^{(2)}\left(k_{0} a\right)-H_{1}^{(2)}\left(k_{0} a\right) \times H_{1}^{(1)}\left(k_{0} a\right)}=-\frac{H_{1}^{(2)^{\prime}}\left(k_{0} a\right)}{4 i} \times \pi k_{0} a
$$


Note that Eqs. (9.1.27) and (9.1.17) of Abramowitz \& Stegun (1965) have been used to simplify the denominators of $\alpha_{1}$ and $\alpha_{2}$. This equation shows that at a given $k_{0}, C_{0 m}$ term will vary with $c$ periodically with period $k_{0} c=\pi$. It will remain the case no matter how large $c$ is. Such behaviour is shown in Figure 5.

At a fixed $c$, when $k_{0}$ varies, $\alpha_{1}$ and $\alpha_{2}$ will also change. Thus, the variation of $C_{0 m}$ with $k_{0} c$ is not exactly periodical. Although the result may still have peaks and troughs with the period of $k_{0} c=\pi$, their values vary with $k_{0}$. More detailed analysis for large $k_{0}$ can be made using (9.2.13) and (9.2.14) of Abramowitz \& Stegun (1965). We can have

$$
\begin{gathered}
\alpha_{1}=-\frac{1}{4} \times H_{1}^{(1)^{\prime}}\left(k_{0} a\right) \times \pi k_{0} a \rightarrow-\frac{1}{4} \times \sqrt{\frac{2}{\pi k_{0} a}} \times i \times e^{i\left(k_{0} a-\frac{3}{4} \pi\right)} \times \pi k_{0} a \sim O\left(\sqrt{k_{0} a}\right) \\
\alpha_{2}=-\frac{1}{4 i} \times H_{1}^{(2)^{\prime}}\left(k_{0} a\right) \times \pi k_{0} a \rightarrow-\frac{1}{4 i} \times \sqrt{\frac{2}{\pi k_{0} a}} \times(-i) \times e^{-i\left(k_{0} a-\frac{3}{4} \pi\right)} \times \pi k_{0} a \sim O\left(\sqrt{k_{0} a}\right)
\end{gathered}
$$

We notice that with the increase of $k_{0}$, both the absolute values of $\alpha_{1}$ and $\alpha_{2}$ will increase, thus $C_{0 m}$ term may decay and become less significant than $A_{n m}$, or the effect of the ice sheet diminishes. However, the decay is proportional to $1 / \sqrt{k_{0} a}$ and therefore it is rather slow. This is consistent with the behaviour shown in Figure 6.
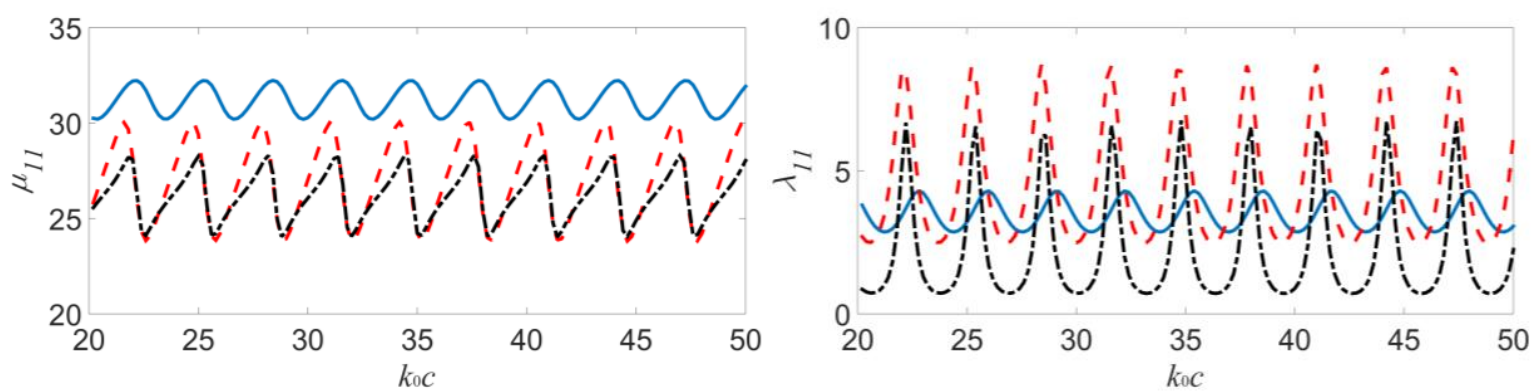

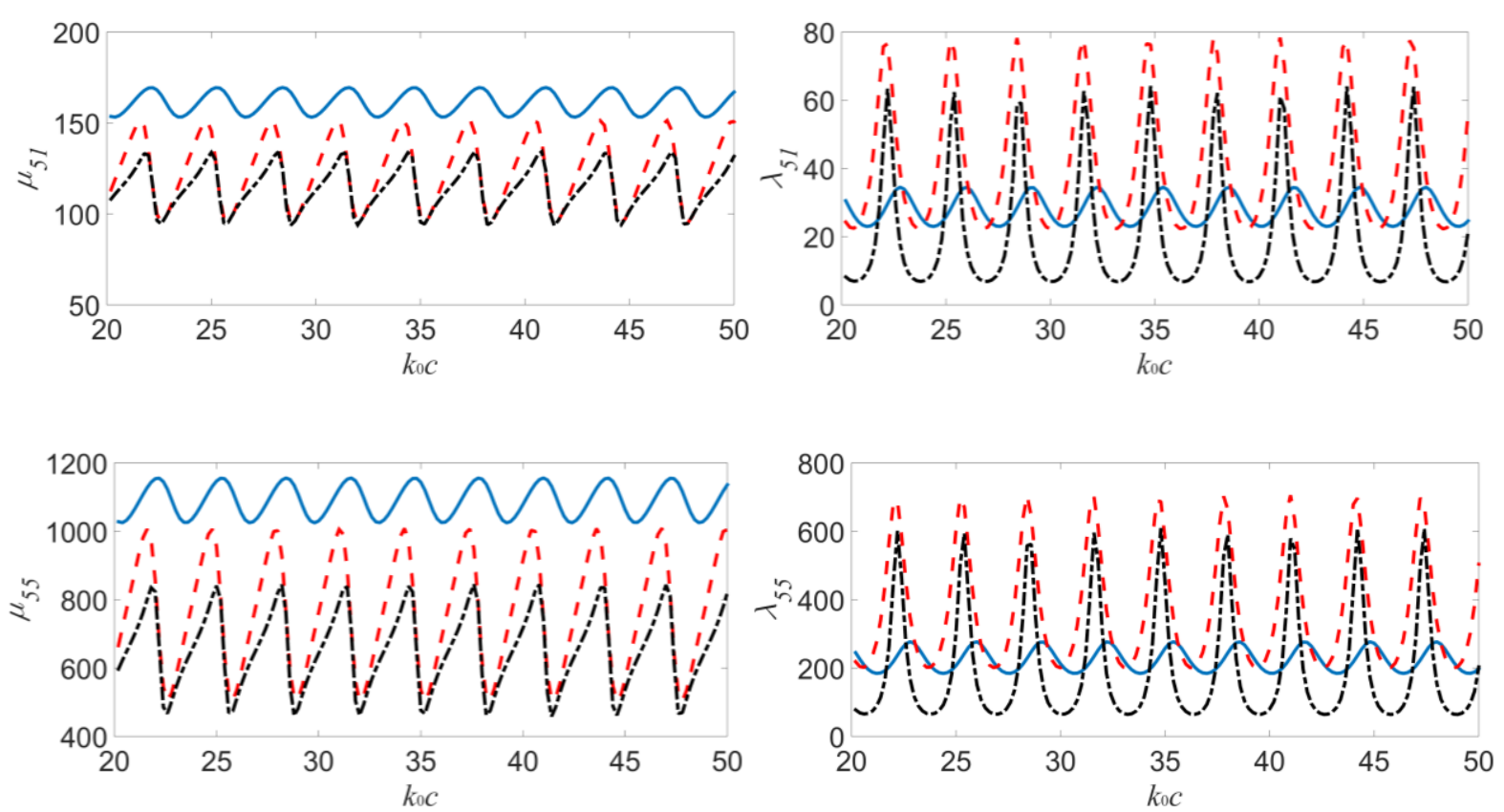

Figure 5. Hydrodynamic coefficients against $k_{0} c$ for fixed $k_{0} a .\left(a=1, h=0.1, H=10, m_{\text {ice }}=\right.$ 0.09, $L=4.5582$ ). Solid lines: $k_{0} a=0.5$; dashed lines: $k_{0} a=1.0$; dotted-dashed lines: $k_{0} a=$ 2.0 .
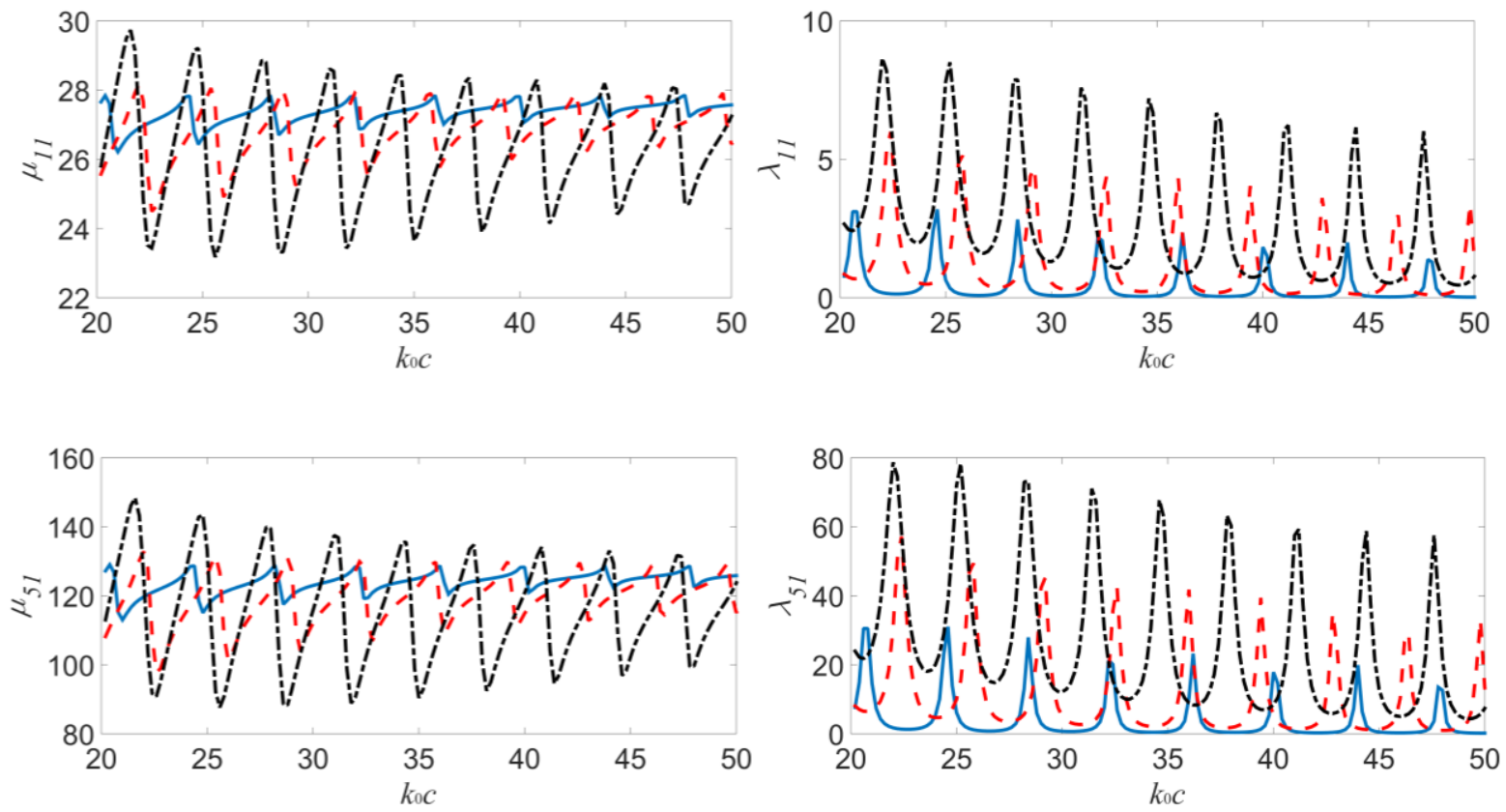

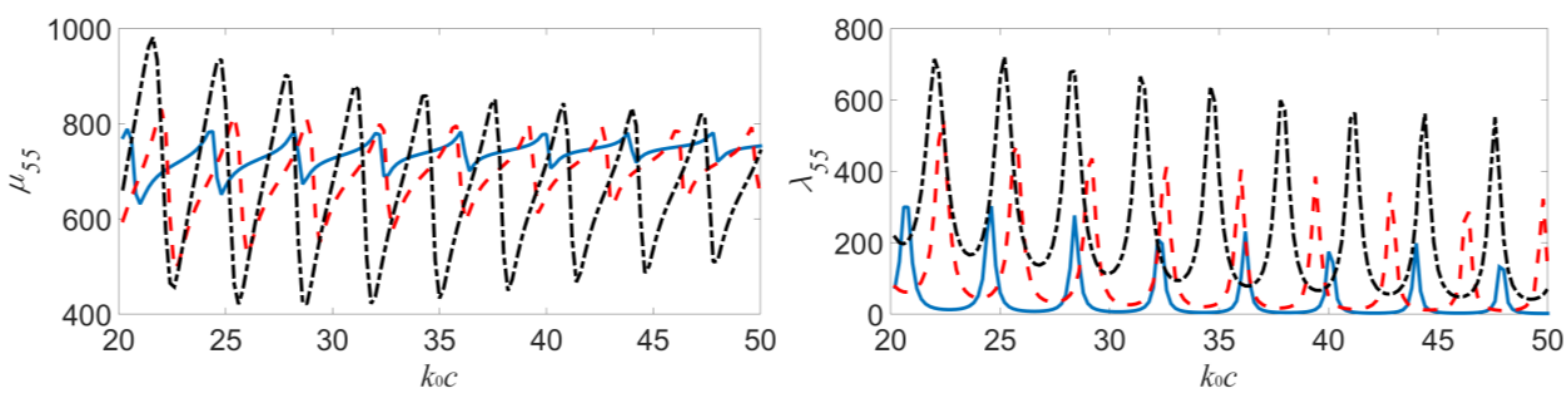

Figure 6. Hydrodynamic coefficients against different $k_{0} c$ for fixed $c .(a=1, h=0.1, H=10$, $m_{i c e}=0.09, L=4.5582$ ). Solid lines: $c=5$; dashed lines: $c=10$; dotted-dashed lines: $c=20$.

The result found here is also consistent with what has been found in the twodimensional problem. Li et al (2017) have shown that for a 2D body of length dimension $a$ in a polynya of width $l$, the results will vary periodically with $k_{0} c$ at a given $k_{0} a$. On the other hand, at large $c / a$, when $k_{0} a$ continues to increase, the results will tend to those in the open water.

The wave run-up due to incident and diffracted wave at the ice edge $(r=c)$ at different wave frequencies $\kappa_{0} c$ and different $c / a$ are shown in Figure 7. Apart from the four frequencies in Figure 3, here we choose two extra frequencies corresponding to peak values of the damping coefficients or exciting forces from Figure 4, namely, $k_{0} a=1.22$ and 2.62 (corresponding $\kappa_{0} c=3.3574$ and 4.2292). It can be seen that for a fixed $c$, with $c / a$ becoming larger, the results of the cases with the cylinder tend to that of body free case. In fact, we may revisit Eqs. (19) and (25). We have $A_{m n}=0$ in this case. When $k_{n} a \rightarrow 0, H_{m}^{(1)^{\prime}}\left(k_{n} a\right) / H_{m}^{(2)^{\prime}}\left(k_{n} a\right) \rightarrow-1$ (see Eqs. (9.1.8), (9.1.9) and (9.1.27) of Abramowitz \& Stegun (1965)). Therefore, we have

$$
\lim _{a \rightarrow 0} \frac{H_{m}^{(2)}\left(k_{n} r_{1}\right) \times H_{m}^{(1)^{\prime}}\left(k_{n} a\right)-H_{m}^{(2)^{\prime}}\left(k_{n} a\right) \times H_{m}^{(1)}\left(k_{n} r_{1}\right)}{H_{m}^{(2)}\left(k_{n} c\right) \times H_{m}^{(1)^{\prime}}\left(k_{n} a\right)-H_{m}^{(2)^{\prime}}\left(k_{n} a\right) \times H_{m}^{(1)}\left(k_{n} c\right)}=\frac{J_{m}\left(k_{n} r_{1}\right)}{J_{m}\left(k_{n} c\right)},
$$


which shows that when $c$ is fixed, the result at $c / a \rightarrow \infty$ tends to that at $c / a=\infty$. This is different with the case when $a$ is fixed and $c / a \rightarrow \infty$.
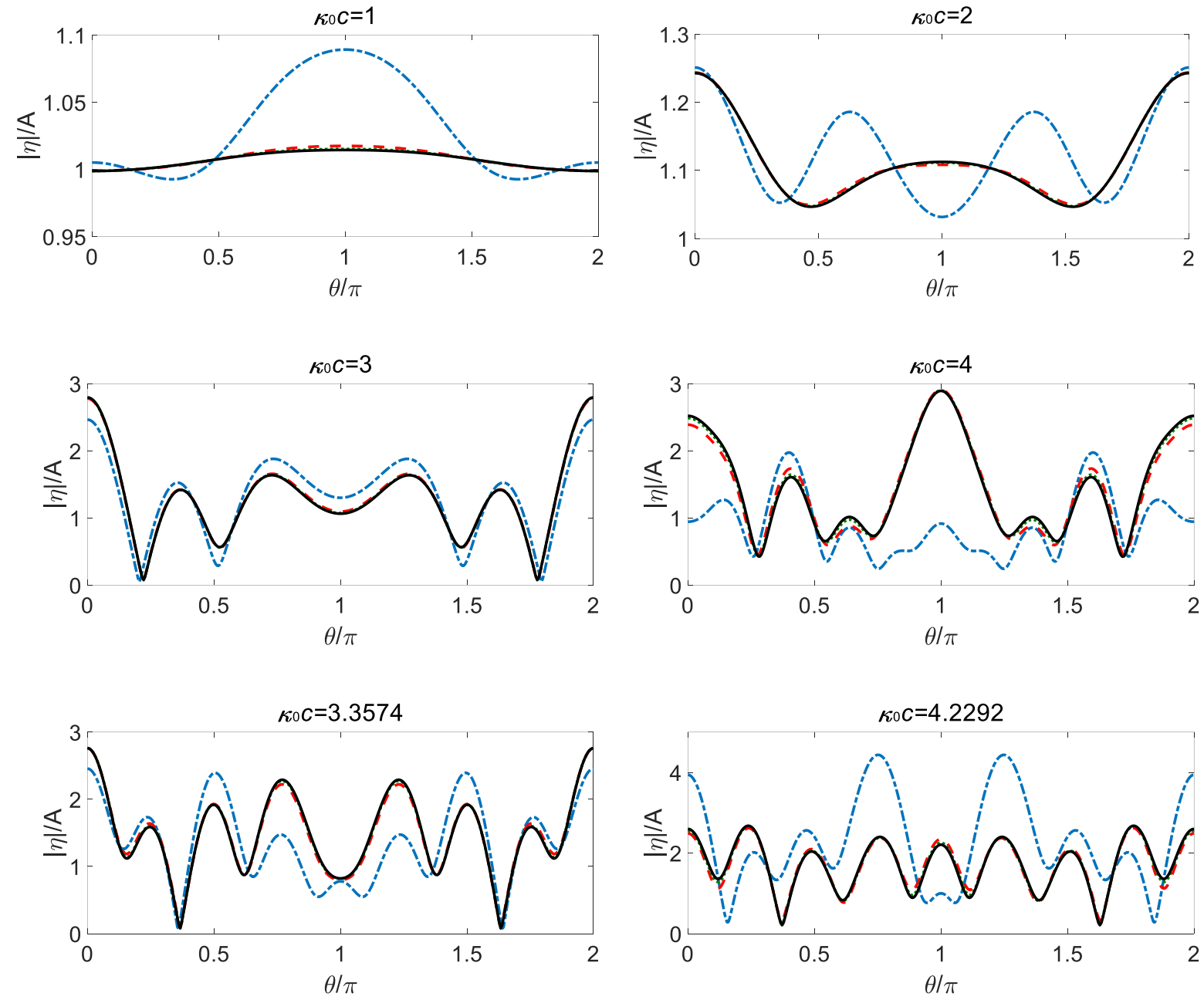

Figure 7. The run-up at the ice edge. $\left(c=1, H=2, m_{i c e}=0.018, L=0.00729\right)$. Dashed-dotted lines: $c / a=5$; dashed lines: $c / a=25$; dotted lines: $c / a=50$; solid lines: $c / a=\infty$.

The wave patterns similar to those in Figure 3, but with a cylinder $(c / a=5)$ centrally located within the polynya are shown in Figure 8. We can find that the introduction of the cylinder makes the flow field more complex, which can also be reflected by the run-up at the ice edge in Figure 7. 

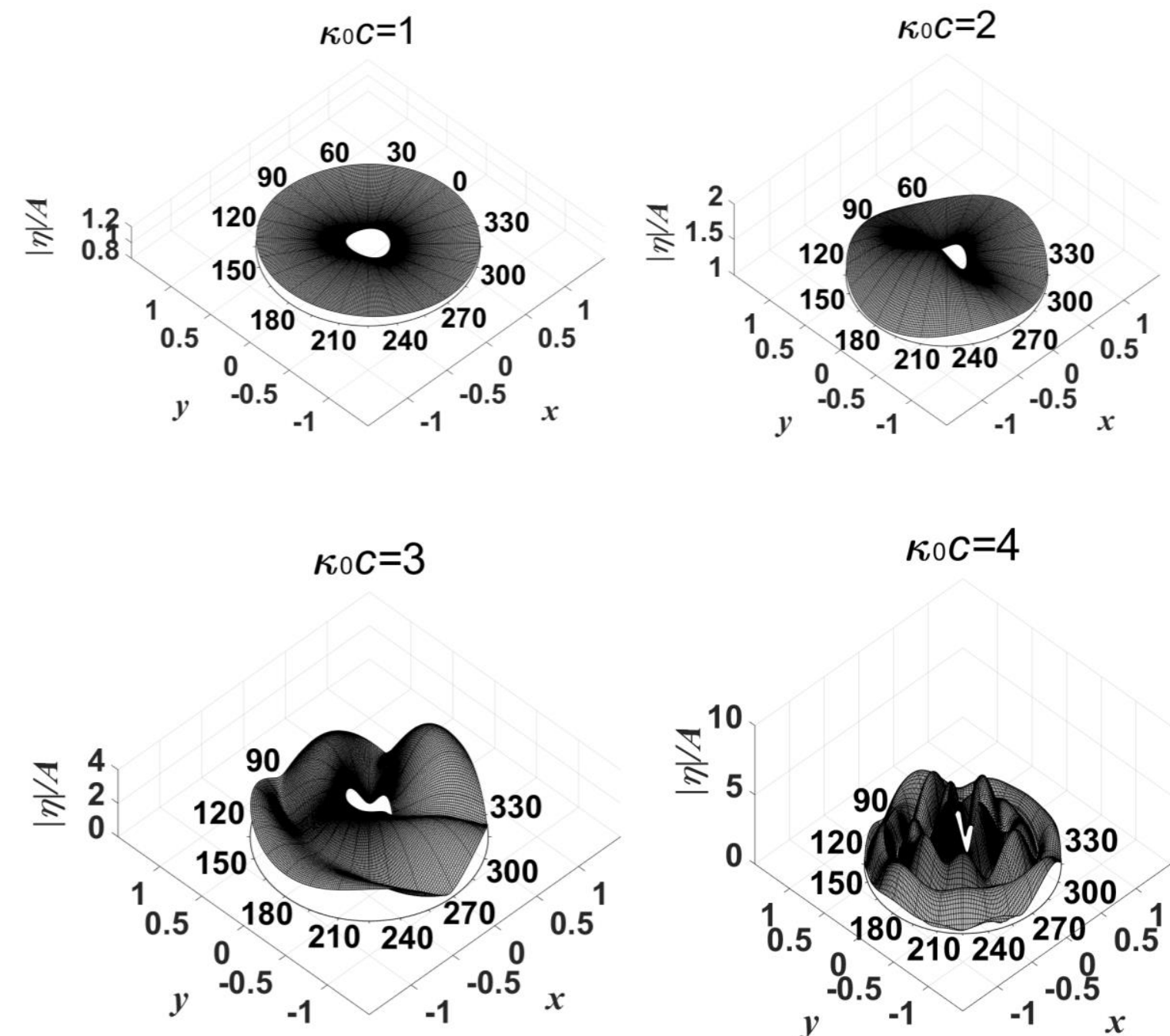

$\kappa 0 C=4$

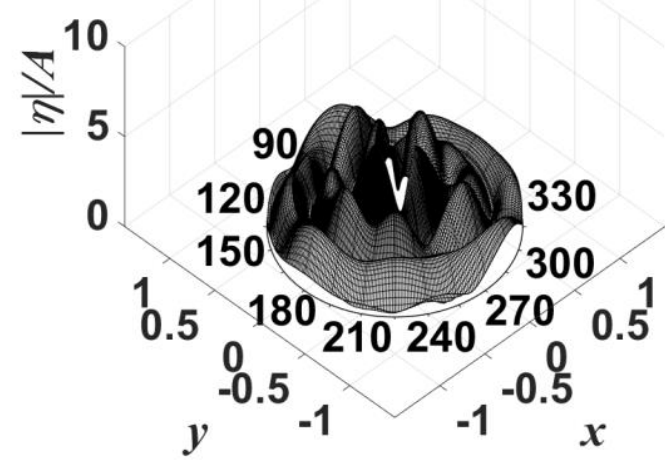

Figure 8 . As in figure 3 but for a cylinder centrally located within the polynya $(c / a=5)$.

(c) An off-centre located cylinder

The effect of the location of the cylinder on the results of hydrodynamic coefficients is now investigated. We first move the cylinder along $x$ axis, or for the fixed $\theta_{0}=0$, change $r_{0}$. This means that the body is closer to the right ice edge. Results corresponding to $c=5$ at $r_{0}=0.5$ and 1.0 are plotted in Figure 9 . It can be seen that when $r_{0}$ increases, the results become more oscillatory. This can be partly explained by the analysis of Li et al (2017) for a similar 2D problem. Their equations show that at given frequency these results oscillate periodically 
not only with respect to the width of the polynya but also to the distance between the body centre and the polynya centre. The second oscillatory term does not appear when the body is at the centre of polynya and therefore the results are less oscillatory.
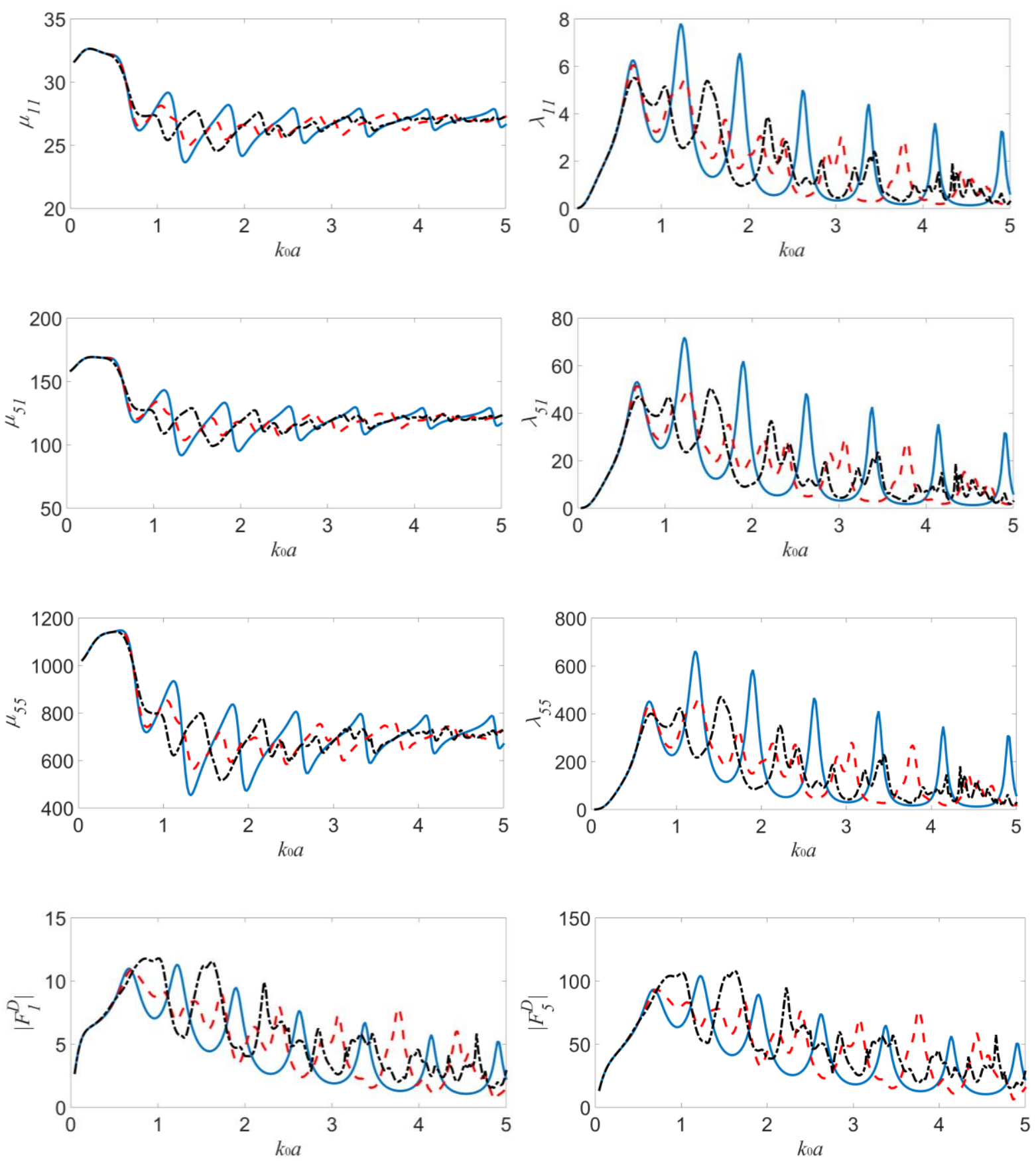

Figure 9. Hydrodynamic coefficients and exciting forces of off-centre located cylinder with different $r_{0}$ at $\theta_{0}=0\left(a=1, c=5, H=10, h=0.1, m_{i c e}=0.09, L=4.5582\right)$. Solid lines: $r_{0}=0$ ; dashed lines: $r_{0}=0.5$; dotted-dashed lines: $r_{0}=1.0$. 
We then consider the case of fixed $r_{0}=0.5$ while $\theta_{0}=\pi / 2$. The results are provided in Figure 10. As discussed after Eq. (7), surge and pitch in the case are in fact the same as sway and roll in $\theta_{0}=0$.
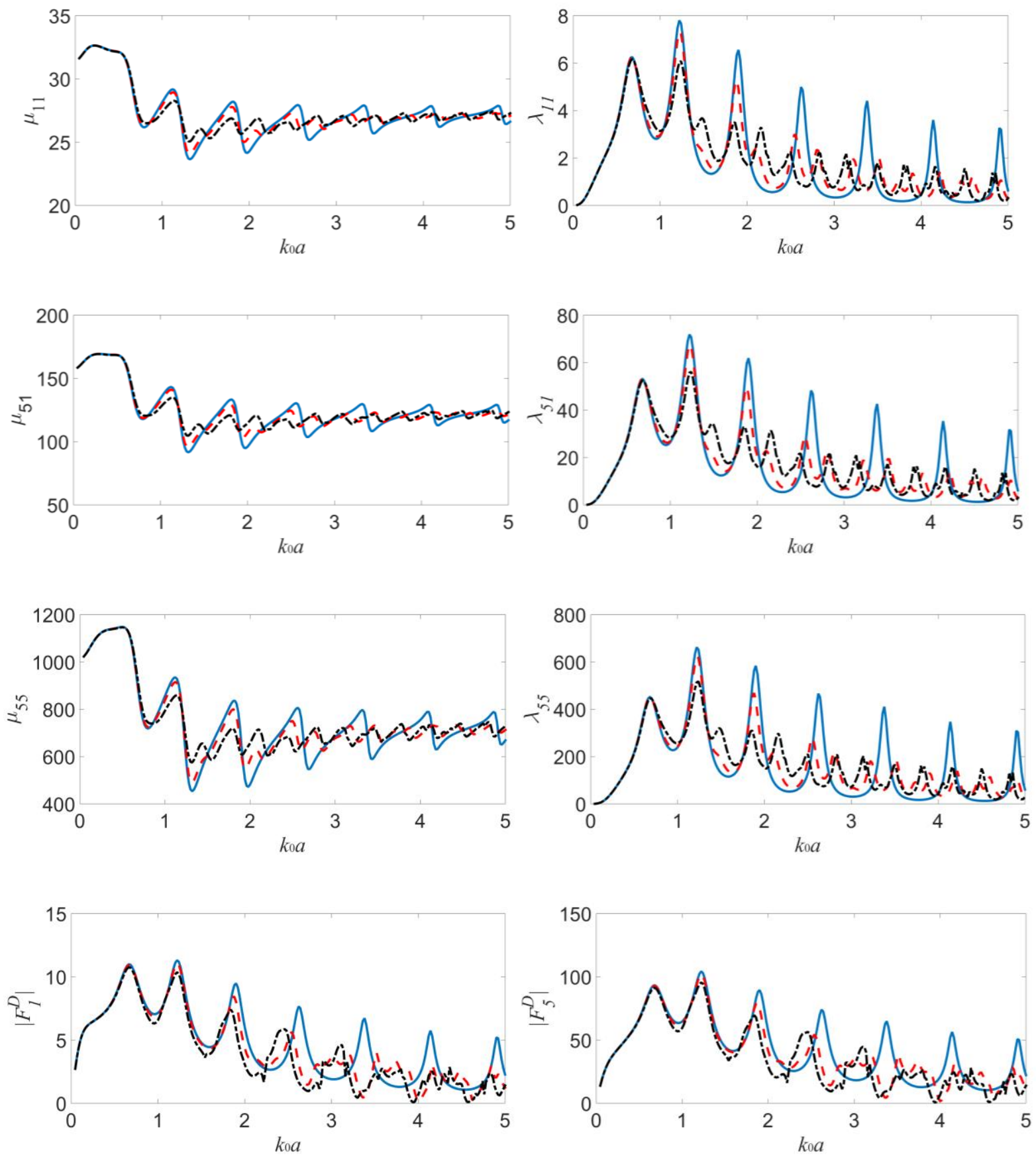
Figure 10. Hydrodynamic coefficients and excitation forces of off-centre located cylinder with different $r_{0}$ at $\theta_{0}=\pi / 2\left(a=1, c=5, H=10, h=0.1, m_{i c e}=0.09, L=4.5582\right)$. Solid lines: $r_{0}=0$; dashed lines: $r_{0}=0.5$; dotted-dashed lines: $r_{0}=1.0$.

For the same fixed $r_{0}$, the results at other $\theta_{0}$ can be obtained from those at $\theta_{0}=0$ and $\theta_{0}=\pi / 2$. In fact, it can be easily established that the surge and sway potential at $\theta_{0}$ can be written as

$$
\begin{aligned}
& \left.\phi_{1}\right|_{\theta_{0}}=\left.\cos \theta_{0} \cdot \phi_{1}\right|_{0}-\left.\sin \theta_{0} \cdot \phi_{2}\right|_{0} \\
& \left.\phi_{2}\right|_{\theta_{0}}=\left.\sin \theta_{0} \cdot \phi_{1}\right|_{0}+\left.\cos \theta_{0} \cdot \phi_{2}\right|_{0}
\end{aligned}
$$

Substituting Eqs. (30a, b) into Eq. (27), we have

$$
\begin{aligned}
& \left.\tau_{11}\right|_{\theta_{0}}=\cos ^{2} \theta_{0} \times\left.\tau_{11}\right|_{0}+\sin ^{2} \theta_{0} \times\left.\tau_{22}\right|_{0}-\sin \theta_{0} \cos \theta_{0} \times\left(\left.\tau_{12}\right|_{0}+\left.\tau_{21}\right|_{0}\right) \\
& \left.\tau_{22}\right|_{\theta_{0}}=\cos ^{2} \theta_{0} \times\left.\tau_{22}\right|_{0}+\sin ^{2} \theta_{0} \times\left.\tau_{11}\right|_{0}+\sin \theta_{0} \cos \theta_{0} \times\left(\left.\tau_{12}\right|_{0}+\left.\tau_{21}\right|_{0}\right)
\end{aligned}
$$

Noticing that $\left.\tau_{12}\right|_{0}=\left.\tau_{21}\right|_{0}=0$ and $\left.\tau_{22}\right|_{0}=\left.\tau_{11}\right|_{\pi / 2},\left.\tau_{11}\right|_{0}=\left.\tau_{22}\right|_{\pi / 2}$, Eqs. (31) become

$$
\begin{aligned}
& \left.\tau_{11}\right|_{\theta_{0}}=\cos ^{2} \theta_{0} \times\left.\tau_{11}\right|_{0}+\sin ^{2} \theta_{0} \times\left.\tau_{11}\right|_{\pi / 2} \\
& \left.\tau_{22}\right|_{\theta_{0}}=\cos ^{2} \theta_{0} \times\left.\tau_{22}\right|_{0}+\sin ^{2} \theta_{0} \times\left.\tau_{22}\right|_{\pi / 2}
\end{aligned}
$$

The behaviour of these results then follows those in Figures 9 and 10. The other hydrodynamic coefficients at any arbitrary $\theta_{0}$ can be obtained in a similar way.

\section{Conclusions}

The wave diffraction and radiation problem of a vertical circular cylinder in a circular polynya surrounded by the ice sheet has been solved based on the elastic thin-plate theory and the velocity potential theory. The semi-analytical method and solution procedure used can account for various ice-edge conditions although 
only the free edge results are provided. The wave elevation within the polynya, hydrodynamic coefficients and exciting forces on the cylinder have been obtained and analysed. Far-field equations for the damping coefficient and exciting force are also derived and have been used to verify the near-field methods. From the extensive calculations undertaken, the following conclusions can be made.

1. The wave within the polynya has a complex pattern. Without the presence of the body, the wave amplitude is quite oscillatory across the polynya, especially as the frequency increases. There are also some parts in the polynya where the amplitude is very small or nearly zero. When the cylinder is introduced in the polynya, the wave pattern within the polynya can be obviously more complex. For the wave run-up on the ice sheet edge, the effect of the cylinder diminishes as its radius diminishes.

2. For the hydrodynamic coefficients, they are quite oscillatory against the radius $c$ of the polynya and the wave number $k_{0}$. In the former case, at sufficiently large $c$, the results oscillate with a period of $k_{0} c=\pi$ at a given $k_{0}$. The oscillation does not decay no matter how large $c$ is, which shows that the result at $c / a \rightarrow \infty$ does not tend to the ice sheet free case. In the latter case of fixed $c$ and varying $k_{0}$, the oscillation also has a period of $k_{0} c=\pi$ when $k_{0}$ is sufficiently large. However, the oscillation decays at a rate of $1 / \sqrt{k_{0} a}$, which suggests that the result will tend to that without the ice sheet as $k_{0}$ increases.

3. For a circular cylinder located off centre within the polynya, the oscillation of the hydrodynamic coefficients depends on not only $k_{0} c$ but also the distance between the cylinder centre and the polynya centre. The hydrodynamic coefficients of the cylinder with its centre at an arbitrary position $\left(r_{0}, \theta_{0}\right)$ within the polynya can be obtained from the combination of the results with cylinder centres at two specific locations $\left(r_{0}, 0\right)$ and $\left(r_{0}, \pi / 2\right)$ respectively. 


\section{Acknowledgements}

The first author is sponsored by the joint scholarship from University College London and China Scholarships Council, to which he is most grateful. The authors also gratefully acknowledge financial support from the Lloyd's Register Foundation (LRF) through the joint centre involving University College London, Shanghai Jiao Tong University, and Harbin Engineering University. The LRF helps protect life and property by supporting engineering-related education, public engagement, and the application of research.

\section{Appendix}

\section{Appendix A. Free edge condition for the ice sheet}

For the free edge, the zero moment and zero Kirchhoff shear force conditions give (Timoshenko \& Woinowsky-Krieger, 1959)

$$
\begin{gathered}
\nabla^{2} w=(1-v)\left[\sin ^{2} \theta \frac{\partial^{2} w}{\partial x^{2}}+\cos ^{2} \theta \frac{\partial^{2} w}{\partial y^{2}}-2 \sin \theta \cos \theta \frac{\partial^{2} w}{\partial x \partial y}\right] \\
\frac{\partial}{\partial n}\left(\nabla^{2} w\right)=-(1-v) \frac{\partial}{\partial s}\left[\left(\cos ^{2} \theta-\sin ^{2} \theta\right) \frac{\partial^{2} w}{\partial x \partial y}+\left(\frac{\partial^{2} w}{\partial y^{2}}-\frac{\partial^{2} w}{\partial x^{2}}\right) \cos \theta \sin \theta\right]
\end{gathered}
$$

where $w$ is the complex vertical displacement of the ice sheet. $\boldsymbol{n}=(\cos \theta, \sin \theta)$ and $s$ is along the arc length of the edge. Noticing

$$
\frac{\partial x}{\partial s}=-\sin \theta, \frac{\partial y}{\partial s}=\cos \theta
$$

and

$$
\frac{1}{R}=\frac{\partial \theta}{\partial s}
$$

where $R$ is the radius of curvature, we have 


$$
\begin{gathered}
\nabla^{2} w=(1-v)\left\{\frac{\partial^{2}}{\partial s^{2}}+\frac{1}{R} \frac{\partial}{\partial n}\right\} w \\
\frac{\partial}{\partial n}\left\{\nabla^{2} w\right\}=-(1-v) \frac{\partial}{\partial s}\left\{\frac{\partial^{2}}{\partial s \partial n}-\frac{1}{R} \frac{\partial}{\partial s}\right\} w
\end{gathered}
$$

The kinematic condition on the ice-covered ice gives $w=\frac{i}{\omega} \phi_{z}$, and therefore $\phi_{z}$ also satisfies Eqs. (A1) and (A2). It should be noticed that $\phi$ is either the individual radiated potential or the incident potential plus the diffracted potential. In this case, the edge conditions can only be used for the first two terms in Eq. (24) partly, which gives

$$
\begin{aligned}
& \nabla^{2} f=\nabla^{2} \frac{\partial \phi_{j}^{(I I)}}{\partial z}-\delta_{j 0} \times \nabla^{2} \frac{\partial \phi_{i n}}{\partial z} \\
& =(1-v)\left\{\frac{\partial^{2}}{\partial s^{2}}+\frac{1}{R} \frac{\partial}{\partial n}\right\} \frac{\partial \phi_{j}^{(I I)}}{\partial z}-\delta_{j 0} \times \nabla^{2} \frac{\partial \phi_{i n}}{\partial z} \\
& \frac{\partial}{\partial n}\left\{\nabla^{2} f\right\}=\frac{\partial}{\partial n}\left\{\nabla^{2} \frac{\partial \phi_{j}^{(I I)}}{\partial z}\right\}-\delta_{j 0} \times \frac{\partial}{\partial n}\left\{\nabla^{2} \frac{\partial \phi_{i n}}{\partial z}\right\} \\
& =-(1-v) \frac{\partial}{\partial s}\left\{\frac{\partial^{2}}{\partial s \partial n}-\frac{1}{R} \frac{\partial}{\partial s}\right\} \frac{\partial \phi_{j}^{(I I)}}{\partial z}-\delta_{j 0} \times \frac{\partial}{\partial n}\left\{\nabla^{2} \frac{\partial \phi_{i n}}{\partial z}\right\}
\end{aligned}
$$

and Eq. (24) can be further expanded as

$$
\left.\begin{array}{l}
-\frac{L}{\rho \omega^{2}} \times \oint_{c_{1}}\left(q \frac{\partial}{\partial n} \nabla^{2} f-\frac{\partial q}{\partial n} \nabla^{2} f-f \frac{\partial}{\partial n} \nabla^{2} q+\frac{\partial f}{\partial n} \nabla^{2} q\right) d s \\
=-\frac{L}{\rho \omega^{2}} \times \oint_{C_{1}}\left\{\begin{array}{l}
\frac{\partial \psi_{n^{\prime} m^{\prime}}}{\partial z} \times\left[-(1-v) \frac{\partial}{\partial s}\left\{\frac{\partial^{2}}{\partial s \partial n}-\frac{1}{R} \frac{\partial}{\partial s}\right\} \frac{\partial \phi_{j}^{(I I)}}{\partial z}-\delta_{j 0} \times \frac{\partial}{\partial n}\left\{\nabla^{2} \frac{\partial \phi_{i n}}{\partial z}\right\}\right] \\
-\frac{\partial}{\partial z}\left(\phi_{j}^{(I I)}-\delta_{j 0} \times \phi_{i n}\right) \times \frac{\partial}{\partial n} \nabla^{2}\left(\frac{\partial \psi_{n^{\prime} m^{\prime}}}{\partial z}\right) \times\left[(1-v)\left\{\frac{\partial^{2}}{\partial s^{2}}+\frac{1}{R} \frac{\partial}{\partial n}\right\} \frac{\partial \phi_{j}^{(I I)}}{\partial z}-\delta_{j 0} \times \nabla^{2} \frac{\partial \phi_{i n}}{\partial z}\right] \\
+\frac{\partial}{\partial n}\left[\frac{\partial}{\partial z}\left(\phi_{j}^{(I I)}-\delta_{j 0} \times \phi_{i n}\right)\right] \times \nabla^{2}\left(\frac{\partial \psi_{n^{\prime} m^{\prime}}}{\partial z}\right)
\end{array}\right\} d s
\end{array}\right\}
$$


Specifically, in the polar system of the present problem, $\boldsymbol{n}$ is equal to $\boldsymbol{r}$, and thus $\frac{\partial}{\partial n}=\frac{\partial}{\partial r}, \frac{\partial}{\partial s}=\frac{\partial}{r \partial \theta}$. For a circle, its radius of curvature $R$ equals its radius $c$. To ensure the normal velocity continuity on the interface $r=c, \frac{\partial \phi_{j}^{(I I)}}{\partial r}$ in the first term of Eq. (21) should be replaced by $\frac{\partial \phi_{j}^{(I)}}{\partial r}$. The coefficients equation based on Eq. (21) can be finally obtained after substituting Eqs. (9), (10), (17), (24) and (A3) into Eq. (21).

\section{Appendix B. Far-field equation for the damping coefficient}

For damping coefficients, from Eq. (27), we have

$$
\frac{2 i}{\rho \omega} \lambda_{k j}=\iint_{S_{b}}\left(\phi_{j} \frac{\partial \phi_{k}^{*}}{\partial n}-\phi_{k}^{*} \frac{\partial \phi_{j}}{\partial n}\right) d S,(j, k=1 \sim 6)
$$

where * indicates the complex conjugate. By applying the Green's second identity, the integration in Eq. (B1) can be converted to the rest of the fluid boundary. Here the integration over the free surface and seabed will disappear when the corresponding boundary conditions are used. To deal with the integration over the ice sheet $S_{I}$, we define functions $f_{1}=\frac{\partial \phi_{j}}{\partial z}$ and $q_{1}=\frac{\partial \phi_{k}^{*}}{\partial z}$, which both satisfy conditions on ice sheet in Eq. (4) and at ice edge in Eq. (A1). Similar to Eq. (24), the surface integral over $S_{I}$ can be transformed into two line integrals respectively along the ice edge and at infinity. Thus, we can obtain

$$
\begin{aligned}
\frac{2 i}{\rho \omega} \lambda_{k j}= & -\iint_{S_{-}}\left(\phi_{j} \frac{\partial \phi_{k}^{*}}{\partial n}-\phi_{k}^{*} \frac{\partial \phi_{j}}{\partial n}\right) d S \\
& =-\iint_{S_{\infty}}\left(\phi_{j} \frac{\partial \phi_{k}^{*}}{\partial r}-\phi_{k}^{*} \frac{\partial \phi_{j}}{\partial r}\right) d S-\iint_{S_{I}}\left(\phi_{j} \frac{\partial \phi_{k}^{*}}{\partial z}-\phi_{k}^{*} \frac{\partial \phi_{j}}{\partial z}\right) d S \\
& =-\iint_{S_{\infty}}\left(\phi_{j} \frac{\partial \phi_{k}^{*}}{\partial r}-\phi_{k}^{*} \frac{\partial \phi_{j}}{\partial r}\right) d S+\frac{L}{\rho \omega^{2}} \times \oint_{c_{1}+C_{\infty}}\left(q_{1} \frac{\partial}{\partial n} \nabla^{2} f_{1}-\frac{\partial q_{1}}{\partial n} \nabla^{2} f_{1}-f_{1} \frac{\partial}{\partial n} \nabla^{2} q_{1}+\frac{\partial f_{1}}{\partial n} \nabla^{2} q_{1}\right) d s
\end{aligned}
$$


Here $\boldsymbol{n}$ is defined as the normal vector into domain $I I$ as in Eq. (24). Using the edge conditions, we have

$$
\begin{aligned}
& q_{1} \frac{\partial}{\partial n} \nabla^{2} f_{1}-\frac{\partial q_{1}}{\partial n} \nabla^{2} f_{1}-f_{1} \frac{\partial}{\partial n} \nabla^{2} q_{1}+\frac{\partial f_{1}}{\partial n} \nabla^{2} q_{1} \\
& =(1-v) \times\left\{\begin{array}{l}
\left.-q_{1} \times \frac{\partial}{\partial s}\left[\frac{\partial^{2} f_{1}}{\partial s \partial n}-\frac{1}{R} \frac{\partial f_{1}}{\partial s}\right]-\frac{\partial q_{1}}{\partial n} \times \frac{\partial^{2} f_{1}}{\partial s^{2}}+f_{1} \times \frac{\partial}{\partial s}\left[\frac{\partial^{2} q_{1}}{\partial s \partial n}-\frac{1}{R} \frac{\partial q_{1}}{\partial s}\right]+\frac{\partial f_{1}}{\partial n} \times \frac{\partial^{2} q_{1}}{\partial s^{2}}-\frac{\partial q_{1}}{\partial n} \times \frac{1}{R} \frac{\partial f_{1}}{\partial n}+\frac{\partial f_{1}}{\partial n} \times \frac{1}{R} \frac{\partial q_{1}}{\partial n}\right\} \\
=(1-v) \times\left\{\begin{array}{l}
-\frac{\partial}{\partial s}\left[q_{1} \times\left(\frac{\partial^{2} f_{1}}{\partial s \partial n}-\frac{1}{R} \frac{\partial f_{1}}{\partial s}\right)\right]-\frac{\partial}{\partial s}\left[\frac{\partial q_{1}}{\partial n} \times \frac{\partial f_{1}}{\partial s}\right]+\frac{\partial}{\partial s}\left[f_{1} \times\left(\frac{\partial^{2} q_{1}}{\partial s \partial n}-\frac{1}{R} \frac{\partial q_{1}}{\partial s}\right)\right]+\frac{\partial}{\partial s}\left[\frac{\partial f_{1}}{\partial n} \times \frac{\partial q_{1}}{\partial s}\right] \\
+\left(\frac{\partial^{2} f_{1}}{\partial s \partial n}-\frac{1}{R} \frac{\partial f_{1}}{\partial s}\right) \times \frac{\partial q_{1}}{\partial s}+\frac{\partial f_{1}}{\partial s} \times \frac{\partial^{2} q_{1}}{\partial s \partial n}-\left(\frac{\partial^{2} q_{1}}{\partial s \partial n}-\frac{1}{R} \frac{\partial q_{1}}{\partial s}\right) \times \frac{\partial f_{1}}{\partial s}-\frac{\partial q_{1}}{\partial s} \times \frac{\partial^{2} f_{1}}{\partial s \partial n}
\end{array}\right\} \\
=(1-v) \times \frac{\partial}{\partial s}\left\{-q_{1} \times\left(\frac{\partial^{2} f_{1}}{\partial s \partial n}-\frac{1}{R} \frac{\partial f_{1}}{\partial s}\right)-\frac{\partial q_{1}}{\partial n} \times \frac{\partial f_{1}}{\partial s}+f_{1} \times\left(\frac{\partial^{2} q_{1}}{\partial s \partial n}-\frac{1}{R} \frac{\partial q_{1}}{\partial s}\right)+\frac{\partial f_{1}}{\partial n} \times \frac{\partial q_{1}}{\partial s}\right\}
\end{array}\right.
\end{aligned}
$$

Therefore

$$
\begin{aligned}
& -\frac{L}{\rho \omega^{2}} \times \int_{0}^{s}\left[q_{1} \frac{\partial}{\partial r}\left(\nabla^{2} f_{1}\right)-\frac{\partial q_{1}}{\partial r}\left(\nabla^{2} f_{1}\right)-f_{1} \frac{\partial}{\partial r}\left(\nabla^{2} q_{1}\right)+\frac{\partial f_{1}}{\partial r}\left(\nabla^{2} q_{1}\right)\right] d s \\
& =-\frac{L \times(1-v)}{\rho \omega^{2}} \times \int_{0}^{s} \frac{\partial}{\partial s}\left\{-q_{1} \times\left(\frac{\partial^{2} f_{1}}{\partial s \partial n}-\frac{1}{R} \frac{\partial f_{1}}{\partial s}\right)-\frac{\partial q_{1}}{\partial n} \times \frac{\partial f_{1}}{\partial s}+f_{1} \times\left(\frac{\partial^{2} q_{1}}{\partial s \partial n}-\frac{1}{R} \frac{\partial q_{1}}{\partial s}\right)+\frac{\partial f_{1}}{\partial n} \times \frac{\partial q_{1}}{\partial s}\right\} d s=0
\end{aligned}
$$

This leads to that only the line integral at infinity left in the second term in Eq. (B2). For the first term of Eq. (B2), it is virtually the same as that without the ice sheet, (e.g. Eq. 6.16; Chapter 7; Mei 1989), apart from that $\kappa_{0}$ is different. We may follow the same procedure. The velocity potential $\phi_{j}$ at infinity can be written as

$$
\phi_{j}=f_{j}(\theta) \times \frac{\cosh \kappa_{0}(H+z)}{\cosh \kappa_{0} H} \times \sqrt{\frac{2}{\pi \kappa_{0} r}} \times e^{i\left(\kappa_{0} r-\pi / 4\right)}
$$

By substituting it into the first term of Eq. (B2), we can obtain

$$
\lambda_{k j}=\left\{\frac{\rho \omega}{\pi} \times\left(\frac{\tanh \kappa_{0} H}{\kappa_{0}}+\frac{H}{\cosh ^{2} \kappa_{0} H}\right)+\frac{4 L}{\pi \omega} \times \kappa_{0}^{4} \times \tanh ^{2} \kappa_{0} H\right\} \times \int_{0}^{2 \pi} f_{k}^{*}(\theta) f_{j}(\theta) d \theta
$$

By further introducing the group velocity of the waves beneath the ice sheet, or 


$$
\begin{aligned}
C_{g} & =\frac{d \omega\left(\kappa_{0}\right)}{d \kappa_{0}}=\frac{4 \kappa_{0}{ }^{4} \frac{L}{\rho} \tanh \kappa_{0} H+\left(g+\frac{L \kappa_{0}{ }^{4}}{\rho}-\frac{m_{i c e} \omega^{2}}{\rho}\right)\left(\tanh \kappa_{0} H+\frac{\kappa_{0} H}{\cosh ^{2} k H}\right)}{2 \omega+\frac{2 m_{i c e} \omega}{\rho} \kappa_{0} \tanh \kappa_{0} H} \\
& =\frac{\pi}{2 \rho \tanh \kappa_{0} H \times\left(1+\frac{m_{i c e} \kappa_{0}}{\rho} \tanh \kappa_{0} H\right)}\left\{\frac{\rho \omega}{\pi}\left(\frac{\tanh \kappa_{0} H}{\kappa_{0}}+\frac{H}{\cosh ^{2} \kappa_{0} H}\right)+\frac{4 L}{\pi \omega} \kappa_{0}^{4} \tanh ^{2} \kappa_{0} H\right\}
\end{aligned}
$$

Eq. (B5) can be further written as

$$
\lambda_{k j}=C_{g} \times \frac{\left(2 \rho \tanh \kappa_{0} H+2 m_{i c e} \kappa_{0} \tanh ^{2} \kappa_{0} H\right)}{\pi} \times \int_{0}^{2 \pi} f_{k}^{*}(\theta) f_{j}(\theta) d \theta
$$

The above expression is for general cases. Specifically, for the present problem, from Eqs. (10) and (11), we have

$$
f_{j}(\theta)=\sum_{m=-\infty}^{\infty} P_{0 m}^{(j)} \times e^{i m \theta} \times \frac{1}{H_{m}^{(1)}\left(\kappa_{0} c\right)} \times e^{-i m \pi / 2}
$$

Eq.(B6) then becomes

$$
\lambda_{k j}=C_{g} \times\left(4 \rho \tanh \kappa_{0} H+4 m_{i c e} \kappa_{0} \tanh ^{2} \kappa_{0} H\right) \times \sum_{m=-\infty}^{\infty} \frac{P_{0 m}^{(j)} \times P_{0 m}^{(k) *}}{H_{m}^{(1)}\left(\kappa_{0} c\right) \times H_{m}^{(1) *}\left(\kappa_{0} c\right)}
$$

\section{Appendix C. Far-field equation for the exciting force}

From Eqs. (6a) and (28) and using the Green's identity, we have

$$
F_{j}^{D}=i \omega \rho \iint_{S_{b}}\left(\phi_{0} \frac{\partial \phi_{j}}{\partial n}-\phi_{j} \frac{\partial \phi_{0}}{\partial n}\right) d S=-i \omega \rho \times\left\{\iiint_{S_{\infty}}\left(\phi_{0} \frac{\partial \phi_{j}}{\partial r}-\phi_{j} \frac{\partial \phi_{0}}{\partial r}\right) d S+\iint_{S_{I}}\left(\phi_{0} \frac{\partial \phi_{j}}{\partial z}-\phi_{j} \frac{\partial \phi_{0}}{\partial z}\right) d S\right\}
$$

Similar to the previous derivation for the damping coefficient, the integration over $S_{I}$ can be converted into a line integral at infinity. Substituting Eqs. (8) and (B4) into the right-hand side of Eq. (C1), and using the method of stationary phase (e.g. Newman,1977), Eq. (C1) can be further written as 


$$
\begin{aligned}
F_{j}^{D} & =\left[\begin{array}{l}
A \frac{i g}{\omega} \times i \kappa_{0} r \times \sqrt{\frac{2}{\pi \kappa_{0} r}} \times e^{i\left(\kappa_{0} r-\pi / 4\right)} \times \int_{0}^{2 \pi}\left\{e^{i \kappa_{0} r \cos \theta} \times(\cos \theta-1) \times f_{j}(\theta)\right\} d \theta \times \int_{-H}^{0}\left(\frac{\cosh \kappa_{0}(H+z)}{\cosh \kappa_{0} H}\right)^{2} d z \\
+\frac{L}{\rho \omega^{2}} \times A \frac{i g}{\omega} \times \kappa_{0}^{2} \tanh ^{2} \kappa_{0} H \times \sqrt{\frac{2}{\pi \kappa_{0} r}} \times e^{i\left(\kappa_{0} r-\pi / 4\right)} \times \int_{0}^{2 \pi} f_{j}(\theta) \times e^{i \kappa_{0} r \cos \theta} \times\left(\begin{array}{l}
i r \kappa_{0}^{3}\left(\cos ^{3} \theta-\cos ^{2} \theta\right) \\
+\kappa_{0}^{2}\left(\cos ^{2} \theta-1\right) \\
+i r \kappa_{0}^{3}(\cos \theta-1)
\end{array}\right) d \theta
\end{array}\right]_{r \rightarrow \infty} \\
& =-i \times \frac{4 A \rho g \tanh \kappa_{0} H}{\omega} \times\left(1+\frac{m_{i c e} \kappa_{0}}{\rho} \tanh \kappa_{0} H\right) \times C_{g} \times f_{j}(\pi)
\end{aligned}
$$

Eq. (C2) is a general formulation for the cases where the incident wave is along the positive $x$ axis or wave direction $\alpha=0$. For other wave direction $\alpha, f_{j}(\pi)$ in Eq. (C2) should be replaced by $f_{j}(\alpha+\pi)$.

For the present problem, substituting Eq. (B7) into Eq. (C2), we can obtain

$$
F_{j}^{D}=-i \times \frac{4 A \rho g \tanh \kappa_{0} H}{\omega} \times\left(1+\frac{m_{i c e} \kappa_{0}}{\rho} \tanh \kappa_{0} H\right) \times C_{g} \times \sum_{m=-\infty}^{\infty} \frac{P_{0 m}^{(j)}}{H_{m}^{(1)}\left(\kappa_{0} c\right)} \times e^{i m \pi / 2}
$$

\section{References}

Abromowitz, M. and Stegun, I.A., 1965. Handbook of mathematical functions. NBS (now NIST).

Andrianov, A.I. and Hermans, A.J., 2005. Hydroelasticity of a circular plate on water of finite or infinite depth. Journal of Fluids and Structures, 20(5), pp.719-733.

Bai, W. and Eatock Taylor, R., 2007. Numerical simulation of fully nonlinear regular and focused wave diffraction around a vertical cylinder using domain decomposition. Applied Ocean Research, 29(1), pp.55-71.

Balmforth, N.J. and Craster, R.V., 1999. Ocean waves and ice sheets. Journal of Fluid Mechanics, 395, pp.89124.

Bennetts, L.G. and Williams, T.D., 2010. Wave scattering by ice floes and polynyas of arbitrary shape. Journal of Fluid Mechanics, 662, pp.5-35.

Brocklehurst, P., Korobkin, A. and Părău, E.I., 2011. Hydroelastic wave diffraction by a vertical cylinder. Philosophical Transactions of the Royal Society of London A: Mathematical, Physical and Engineering Sciences, 369(1947), pp.2832-2851.

Chau, F.P. and Eatock Taylor, R., 1992. Second-order wave diffraction by a vertical cylinder. Journal of Fluid Mechanics, 240, pp.571-599.

Chen, X.B., Molin, B. and Petitjean, F., 1995. Numerical evaluation of the springing loads on tension leg platforms. Marine Structures, 8(5), pp.501-524. 
Chung, H. and Fox, C., 2002. Calculation of wave-ice interaction using the Wiener-Hopf technique. New Zealand J. Math, 31(1), pp.1-18.

Drake, K.R., Eatock Taylor, R. and Matsui, T., 1984, August. Drift of an articulated cylinder in regular waves. In Proceedings of the Royal Society of London A: Mathematical, Physical and Engineering Sciences (Vol. 394, No. 1807, pp. 363-385). The Royal Society.

Eatock Taylor, R. and Hung, S.M., 1985. Wave drift enhancement effects in multi column structures. Applied Ocean Research, 7(3), pp.128-137.

Eatock Taylor, R. and Hung, S.M., 1987a. Wave drift enhancement effects for semisubmersible and TLP systems. Journal of Offshore Mechanics and Arctic Engineering, 109(4), pp.327-334.

Eatock Taylor, R. and Hung, S.M., 1987b. Second order diffraction forces on a vertical cylinder in regular waves. Applied Ocean Research, 9(1), pp.19-30.

Eatock Taylor, R., Hung, S.M. and Chau, F.P., 1989. On the distribution of second order pressure on a vertical circular cylinder. Applied Ocean Research, 11(4), pp.183-193.

Eatock Taylor, R. and Sincock, P., 1989. Wave upwelling effects in TLP and semisubmersible structures. Ocean Engineering, 16(3), pp.281-306.

Evans, D.V. and Porter, R., 1997. Trapped modes about multiple cylinders in a channel. Journal of Fluid Mechanics, 339, pp.331-356.

Faltinsen, O.M., 1990. Wave loads on offshore structures. Annual Review of Fluid Mechanics, 22(1), pp.35-56.

Fox, C. and Squire, V.A., 1990. Reflection and transmission characteristics at the edge of shore fast sea ice. Journal of Geophysical Research: Oceans, 95(C7), pp.11629-11639.

Fox, C. and Squire, V.A., 1994. On the oblique reflexion and transmission of ocean waves at shore fast sea ice. Philosophical Transactions of the Royal Society of London A: Mathematical, Physical and Engineering Sciences, 347(1682), pp.185-218.

Huang, J.B. and Eatock Taylor, R., 1996. Semi-analytical solution for second-order wave diffraction by a truncated circular cylinder in monochromatic waves. Journal of Fluid Mechanics, 319, pp.171-196.

Kim, M.H. and Yue, D.K., 1988. The nonlinear sum-frequency wave excitation and response of a tension-leg platform.

Lighthill, J., 1986. Fundamentals concerning wave loading on offshore structures. Journal of Fluid Mechanics, 173, pp.667-681.

Linton, C.M. and Evans, D.V., 1990. The interaction of waves with arrays of vertical circular cylinders. Journal of Fluid Mechanics, 215, pp.549-569.

Linton, C.M. and McIver, P., 1996. The scattering of water waves by an array of circular cylinders in a channel. Journal of Engineering Mathematics, 30(6), pp.661-682.

Li, Z.F., Shi, Y.Y. and Wu, G.X., 2018. Interaction of waves with a body floating on polynya between two semiinfinite ice sheets. Journal of Fluids and Structures, 78, pp.86-108.

Li, Z.F., Shi, Y.Y. and Wu, G.X., 2017. Interaction of wave with a body floating on a wide polynya. Physics of Fluids, 29(9), p.097104.

Malenica, S̆., Eatock Taylor, R. and Huang, J.B., 1999. Second-order water wave diffraction by an array of vertical cylinders. Journal of Fluid Mechanics, 390, pp.349-373.

Malenica, Š., Korobkin, A.A. and Gueret, R., 2003, January. Water wave diffraction by vertical circular cylinder in partially frozen sea. In 18th International Workshop on Water Waves and Floating Bodies in Le Croisic, France. 
Maniar, H.D. and Newman, J.N., 1997. Wave diffraction by a long array of cylinders. Journal of Fluid Mechanics, 339, pp.309-330.

Ma, Q.W., Wu, G.X. and Eatock Taylor, R., 2001. Finite element simulations of fully non-linear interaction between vertical cylinders and steep waves. Part 2: numerical results and validation. International Journal for Numerical Methods in Fluids, 36(3), pp.287-308.

Mei, C. C., 1989. The applied dynamics of ocean surface waves. World Scientific.

Meylan, M. and Squire, V.A., 1994. The response of ice floes to ocean waves. Journal of Geophysical Research: Oceans, 99(C1), pp.891-900.

Meylan, M.H. and Squire, V.A., 1996. Response of a circular ice floe to ocean waves. Journal of Geophysical Research: Oceans, 101(C4), pp.8869-8884.

Molin, B., 1979. Second-order diffraction loads upon three-dimensional bodies. Applied Ocean Research, 1(4), pp.197-202.

Newman, J. N., 1977. Marine hydrodynamics. MIT press.

Ren, K., Wu, G.X. and Thomas, G.A., 2016. Wave excited motion of a body floating on water confined between two semi-infinite ice sheets. Physics of Fluids, 28(12), p.127101.

Sahoo, T., Yip, T.L. and Chwang, A.T., 2001. Scattering of surface waves by a semi-infinite floating elastic plate. Physics of Fluids, 13(11), pp.3215-3222.

Smith, M.J., Meylan, M.H. and McPhedran, R.C., 2011. Scattering by cavities of arbitrary shape in an infinite plate and associated vibration problems. Journal of Sound and Vibration, 330(16), pp.4029-4046.

Squire, V.A., Dugan, J.P., Wadhams, P., Rottier, P.J. and Liu, A.K., 1995. Of ocean waves and sea ice. Annual Review of Fluid Mechanics, 27(1), pp.115-168.

Squire, V.A., 2007. Of ocean waves and sea-ice revisited. Cold Regions Science and Technology, 49(2), pp.110133.

Squire, V.A., 2011. Past, present and impendent hydroelastic challenges in the polar and subpolar seas. Philosophical Transactions of the Royal Society of London A: Mathematical, Physical and Engineering Sciences, 369(1947), pp.2813-2831.

Sturova, I.V., 2015. Radiation of waves by a cylinder submerged in water with ice floe or polynya. Journal of Fluid Mechanics, 784, pp.373-395.

Timoshenko, S., \& Woinowsky-Krieger, S., 1959. Theory of Plates and Shells, 2nd ed. McGraw-Hill, Singapore.

Tkacheva, L.A., 2001. Scattering of surface waves by the edge of a floating elastic plate. Journal of Applied Mechanics and Technical Physics, 42(4), pp.638-646.

Tkacheva, L.A., 2004. The diffraction of surface waves by a floating elastic plate at oblique incidence. Journal of Applied Mathematics and Mechanics, 68(3), pp.425-436.

Wang, C.D. and Meylan, M.H., 2004. A higher-order-coupled boundary element and finite element method for the wave forcing of a floating elastic plate. Journal of Fluids and Structures, 19(4), pp.557-572.

Wang, C.Z. and Wu, G.X., 2007. Time domain analysis of second-order wave diffraction by an array of vertical cylinders. Journal of Fluids and Structures, 23(4), pp.605-631.

Wang, C.Z. and Wu, G.X., 2010. Interactions between fully nonlinear water waves and cylinder arrays in a wave tank. Ocean Engineering, 37(4), pp.400-417.

Yeung, R.W. and Sphaier, S.H., 1989. Wave-interference effects on a truncated cylinder in a channel. Journal of Engineering Mathematics, 23(2), pp.95-117. 
Zhou, B.Z. and Wu, G.X., 2015. Resonance of a tension leg platform exited by third-harmonic force in nonlinear regular waves. Philosophical Transactions of the Royal Society of London A: Mathematical, Physical and Engineering Sciences, 373(2033), p.20140105. 